\title{
Neutrino flavor as a test of the explosion mechanism of core-collapse supernovae
}

\author{
Nitsan Bar* \\ Weizmann Institute of Science, Rehovot 7610001, Israel \\ Kfir Blum ${ }^{\dagger}$ \\ Weizmann Institute of Science, Rehovot 7610001, Israel \\ and Theory department, CERN, CH-1211 Geneve 23, Switzerland \\ Guido D'Amico \\ Theory department, CERN, CH-1211 Geneve 23, Switzerland \\ and Stanford Institute for Theoretical Physics, Stanford University, Stanford, California 94306, USA \\ (Received 21 January 2019; published 6 June 2019)

\begin{abstract}
We study the ratio of neutrino-proton elastic scattering to inverse beta decay event counts, measurable in a scintillation detector like JUNO, as a key observable for identifying the explosion mechanism of a galactic core-collapse supernova. If the supernova is not powered by the core but rather, e.g., by collapseinduced thermonuclear explosion, then a prolonged period of accretion-dominated neutrino luminosity is predicted. Using 1D numerical simulations, we show that the distinct resulting flavor composition of the neutrino burst can be tested in JUNO with high significance, overcoming theoretical uncertainties in the progenitor star profile and equation of state.
\end{abstract}

DOI: $10.1103 /$ PhysRevD.99.123004

\section{INTRODUCTION}

How do core-collapse supernovae (CCSNe) explode? A popular belief is that the explosion follows the delayed neutrino mechanism $(\mathrm{D} \nu \mathrm{M})$ [1-4]. In the $\mathrm{D} \nu \mathrm{M}$, explosion is hypothesized to start a fraction of a second after core-collapse with the neutrino-assisted revival of a stalled core bounce shock, about $\sim 100 \mathrm{~km}$ above the surface of a proto-neutron star (PNS). This paradigm, however, has not yet been fully corroborated by numerical simulations. Numerically converged spherically symmetric (1D) $D \nu M$ simulations of iron-core stars consistently fail to explode [5]. Simulations in 2D and 3D pose an extreme numerical challenge and, as a result, present somewhat controversial conclusions. Numerical convergence has not yet been demonstrated for simulations that produce explosions. Typically, numerical noise is introduced in order to seed

\footnotetext{
*nitsan.bar@weizmann.ac.il

†fir.blum@cern.ch

"guido.damico@cern.ch
}

Published by the American Physical Society under the terms of the Creative Commons Attribution 4.0 International license. Further distribution of this work must maintain attribution to the author(s) and the published article's title, journal citation, and DOI. nonspherical instabilities, ${ }^{1}$ instead of deriving these instabilities from the actual physical conditions during collapse $[10,11]$. To date, a self-consistent exploding simulation that reproduces the energetics of, e.g., SN1987A, has not been reported. Success could be just around the corner [12]; nevertheless, it may be useful to keep an open mind to the possibility that the $\mathrm{D} \nu \mathrm{M}$ fails.

While not yet at the same level of sophistication as $\mathrm{D} \nu \mathrm{M}$ simulations, there are competing alternative models to explain CCSNe [13]. Some of these proposals, including the MHD mechanism of [14-18], the acoustic mechanism of [19], or the jet activity suggested in [20], share with the $\mathrm{D} \nu \mathrm{M}$ the attempt to couple a small fraction (of order a percent) of the gravitational binding energy release of the collapsing core into an outward explosion of the envelope. Another, qualitatively different model is collapse-induced thermonuclear explosion (CITE) [21-25]. In CITE, the explosion occurs $\mathcal{O}(10) \mathrm{sec}$ after core collapse due to thermonuclear detonation, triggered some $\sim 10^{4} \mathrm{~km}$ out of the core when a rotation-induced accretion shock traverses an explosive layer in the envelope. Unlike the $\mathrm{D} \nu \mathrm{M}$, the source of energy here is not the gravitational energy of the core. Instead, the observed explosion kinetic energies

\footnotetext{
${ }^{1}$ See, e.g., the discussion in App. 1 and Sec. II.1 of [6]; in Sec. V of [7]; in Sec. II of [8]; or in Sec. IV of [9] and references there.
} 
of CCSNe, $E_{K} \sim 10^{51}-10^{52} \mathrm{erg}$, are reproduced by the $\sim \mathrm{MeV}$ nuclear binding energy per nucleon, released in burning a few $M_{\odot}$ of the progenitor star [26]. On the one hand, the initial conditions required for CITE have not yet been demonstrated in self-consistent stellar evolution codes [27]. On the other hand, converged 2D numerical simulations of CITE with a wide variety of initial conditions explode stars with correct energetics [25,26].

The Rosetta Stone of CCSNe is the neutrino burst. The neutrino burst of SN1987A [28,29] was consistent with the broad characteristics of core-collapse [30-34]. However, the sparse data were not enough to identify the explosion mechanism: CITE and the D $2 \mathrm{M}$ are equally compatible with the neutrino burst of SN1987A [35]. The situation would change with the occurrence of a neutrino burst from a galactic CCSN [36], that would trigger thousands of events in terrestrial detectors (see $[37,38]$ for recent reviews). Still, core-collapse is complicated and the neutrino burst exhibits temporal behavior reflecting the density, composition, and internal rotation profile, that vary between different progenitor stars [39-41]. It is therefore important to identify robust signatures of the main physical phenomena in the explosion. In this work we study neutrino flavor-specifically the $\bar{\nu}_{e}$ fraction of the total neutrino flux-as a key diagnostic of a CCSN neutrino burst. The physics point we wish to study hinges on the neutrino source on times larger than a few hundred milliseconds postbounce.

In all models, core bounce is immediately followed by a quasistationary shock forming at $r_{\text {shock }} \lesssim 100 \mathrm{~km}$. Matter falls through this shock with an accretion rate $\dot{M} \sim(0.1-1) M_{\odot} /$ s. During this accretion phase, ${ }^{2}$ the neutrino source contains a component of $\nu_{e}$ and $\bar{\nu}_{e}$ coming from $e^{+} e^{-}$annihilation $^{3}$ and nucleon conversion in an optically thin region above the PNS neutrinosphere and below the shock. This accretion luminosity is augmented by PNS cooling luminosity of neutrinos of all flavors. Overall, numerical simulations find a source flavor ratio of

$$
\frac{L_{\bar{\nu}_{e}}}{L_{\nu_{x}}}=f_{\bar{e}}, \quad \frac{L_{\nu_{e}}}{L_{\nu_{x}}}=f_{e}, \quad f_{\bar{e}} \approx f_{e} \gtrsim 2,
$$

during the accretion phase, where $L_{\nu_{x}}=L_{\nu_{\mu}}=L_{\nu_{\tau}}=$ $L_{\bar{\nu}_{\mu}}=L_{\bar{\nu}_{\tau}}$.

The initial accretion phase is the same in CITE and in the $\mathrm{D} \nu \mathrm{M}$. What comes next, however, could be significantly different. In CITE, the same initial period of accretion

\footnotetext{
${ }^{2}$ The accretion phase is preceded by a short ( $\sim$ ms duration) burst of $\nu_{e}$ from the deleptonization of the core. This initial $\nu_{e}$ burst is common to all models and we do not discuss it further, focusing instead on the postbounce dynamics.

${ }^{3}$ The cross section for $e^{+} e^{-} \rightarrow \nu_{e} \bar{\nu}_{e}$ is $\sim 4.5$ times larger than that for $e^{+} e^{-} \rightarrow \nu_{x} \bar{\nu}_{x}$ in the energy range of interest $\left(E_{e^{ \pm}}<100 \mathrm{MeV}\right)$.
}

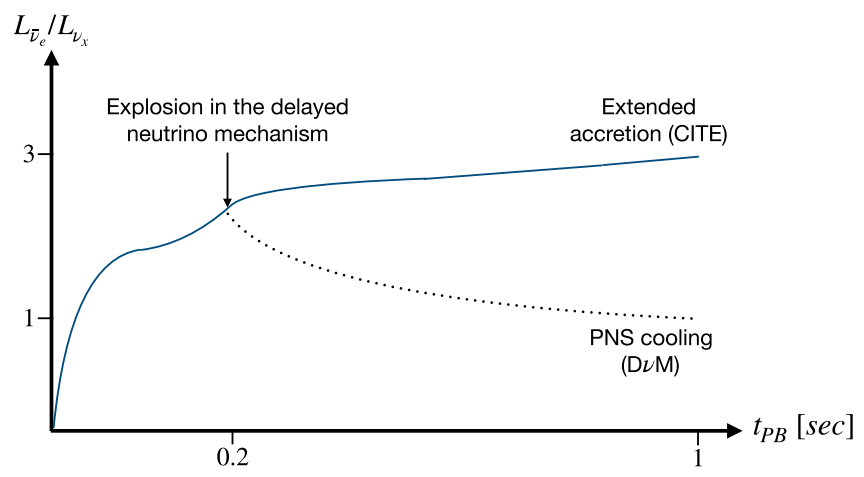

FIG. 1. Cartoon of luminosity ratio vs postbounce time. For $t_{\mathrm{PB}} \lesssim 0.2 \mathrm{sec}$ different explosion mechanisms agree. Then, CITE predicts an extended period of accretion that may last for several seconds. On the other hand, around $t_{\mathrm{PB}} \sim$ 0.2-0.4 sec, the $\mathrm{D} \nu \mathrm{M}$ predicts that the explosion should start, after which neutrino emission is dominated by PNS cooling. (Note that residual accretion in the $\mathrm{D} \nu \mathrm{M}$ could increase $L_{\bar{\nu}_{e}} / L_{\nu_{x}}$ above the pure PNS cooling limit, shown by the dotted line.) In this work we focus on the observational identification of $L_{\bar{\nu}_{e}} / L_{\nu_{x}}>1$ during $t_{\mathrm{PB}} \gtrsim 0.2 \mathrm{sec}$.

luminosity at $t<1 \mathrm{sec}$ continues on to $t>1 \mathrm{sec}$ and can last up to a few seconds, much longer than is expected in the $\mathrm{D} \nu \mathrm{M}$. In some cases, especially for strong explosions with $E_{K} \gtrsim 10^{51} \mathrm{erg}$, the extended accretion phase can lead to the direct formation of a black hole (BH) $[25,35]{ }^{4}$ $\mathrm{BH}$ formation abruptly cuts off the neutrino emission. However, if the star is rotating (as is needed for CITE), an accretion disk must form within $t \lesssim 10 \mathrm{sec}$. Further neutrino emission comes from accretion disk luminosity, that is again dominated by $\nu_{e}$ and $\bar{\nu}_{e}$ at the source [35, 43-45]. This scenario leads to $f_{\bar{e}} \approx f_{e} \gtrsim 2$, most likely rising with time, throughout the neutrino burst.

In the $\mathrm{D} \nu \mathrm{M}$, within a fraction of a second from core bounce, the stalled shock above the PNS must transition into an outward explosion, if the star is to explode at all. The explosion strips off much of the accreting material above the neutrinosphere. From this point on, the neutrino source in the $\mathrm{D} \nu \mathrm{M}$ is dominated by the cooling PNS, emitting a comparable flux of neutrinos of all flavors. Importantly, the $\mathrm{D} \nu \mathrm{M}$ predicts that $f_{\bar{e}}$ and $f_{e}$ decrease in time. In the limit of bare PNS cooling luminosity, the flavor ratio approaches $f_{\bar{e}} \sim f_{e} \sim 1$ once the explosion gets on its way. More realistically, residual accretion could continue even while the star is exploding, leading to $f_{e}$ and $f_{\bar{e}}$ slightly larger than unity [7] but still $f_{e}, f_{\bar{e}}<2$, not rising with time.

A cartoon of $L_{\bar{\nu}_{e}} / L_{\nu_{x}}$ during the first $1 \mathrm{sec}$ postbounce is shown in Fig. 1.

\footnotetext{
${ }^{4}$ Reference [27] points out that the $E_{K} \sim 1.9 \times 10^{51} \mathrm{erg}$ inferred for SNR Kes 73 (G27.4+0.0) [42], which hosts a NS remnant, may pose a problem for CITE.
} 
Can the neutrino flavor at the source be measured by terrestrial detectors? the answer is yes, for specific flavor observables, given the appropriate detector, and assuming that some theoretical features of neutrino oscillations are understood. Consider the ratio:

$$
R=\frac{\text { number of } \mathrm{pES} \text { events }}{\text { number of IBD events }}
$$

measurable in a scintillation detector like JUNO [46], currently under construction (see also the LENA [47] proposal). Here, $\mathrm{pES}$ denotes neutrino-proton elastic scattering, $\nu+p \rightarrow \nu+p$, sensitive to all neutrino species, ${ }^{5}$ and IBD stands for inverse beta decay, $\bar{\nu}_{e}+p \rightarrow e^{+}+n$. Obviously, $R$ measures neutrino flavor at Earth. Connecting this information to flavor at the source is a nontrivial task, requiring knowledge of neutrino propagation and detection cross sections. Even once this is achieved, theoretical uncertainties in the source itself could affect the interpretation of the results. Our goal in this work is to study both sources of uncertainty, those related to propagation and detection and those related to source modeling. The end result of our analysis is that with a realistic treatment of all of these effects, in the event of a galactic CCSN JUNO could identify $f_{\bar{e}} \gtrsim 2$ (predicted in CITE throughout the neutrino burst for $t \gtrsim 0.3 \mathrm{sec}$ postbounce) from $f_{\bar{e}} \sim 1$ (predicted in the simple PNS cooling limit, which could characterize the $\mathrm{D} \nu \mathrm{M}$ once explosion starts), with high significance.

There are several caveats. The most important caveat is due to neutrino self-induced oscillations at the source, which is an open theoretical problem. We will assume that self-induced oscillations do not play an important role in the neutrino propagation. This assumption appears realistic for the prolonged accretion scenario (CITE) [48-50], but it may be less realistic for the $\mathrm{D} \nu \mathrm{M}$. A second caveat is the theoretical uncertainty on the pES cross section. There is a proposal to eliminate this problem [51], possibly in JUNO itself, and we assume that this program succeeds. We find that all of the other issues, including the theoretical uncertainty in progenitor star properties and EoS, are under control, in the sense that accretion-dominated neutrino emission leads to robust predictions and can be distinguished from PNS cooling.

CCSN neutrino flavor and the relevant capabilities of scintillation detectors were studied in a number of previous works [7,52-63]. References [52,53,56] highlighted the importance of $\mathrm{pES}$ as a probe of the total neutrino flux at the source. Reference [55] analyzed the prospects for measuring the spectrum of CCSN $\nu_{e}$ via various subleading reactions in scintillation detectors (following [54] which considered the measurement of $\nu_{e}$ in water Cherenkov detectors doped with Gadolinium). Still focusing on $\nu_{e}$,

\footnotetext{
${ }^{5}$ Almost equally; see Sec. III B and Appendix B.
}

Ref. [56] took a similar approach to ours, in that the neutrino spectra were based on numerical simulations [6] and the need to fit for an a priori unknown spectral shape was accounted for. The neutronization, accretion, and PNS cooling phases of the neutrino burst were analyzed with respect to the capabilities of the water Cherenkov detectors Super-K [64] and Hyper-K [65] and the liquid Ar detector DUNE [66]. References [57-61] presented likelihood analyses of spectral reconstruction in water Cherenkov detectors, assuming analytic equipartition (PNS cooling) source models.

Reference [62] considered the accuracy by which JUNO could reconstruct the mean energy per neutrino $\left\langle E_{\nu_{\alpha}}\right\rangle$ and the total energy carried by neutrinos of each flavor $E_{\nu_{\alpha}}^{\text {tot }}$, assuming an analytic equipartition source model. Accretion-dominated neutrino emission, in which energy equipartition is violated, was not studied. In comparison, we use numerical simulations aiming to go beyond an illustration of the detector capabilities, and study the actual predicted neutrino burst.

Reference [63] used 2D CCSN simulations to analyze the expected signals in Super-K, Hyper-K, and DUNE, focusing on the relation between the progenitor core structure and the neutrino signal intensity during the accretion phase. Reference [7] used 1D and 2D CCSN simulations $[12,67,68]$ to analyze the expected signals in Super-K, DUNE, JUNO, and IceCube. The dependence on progenitor mass in the range $9-21 M_{\odot}$ was studied. The characteristics of the accretion phase were discussed, emphasizing the $\bar{\nu}_{e}$ and $\nu_{e}$ dominance, and arguing that the cessation of the accretion component due to the explosion would provide key information to test the $\mathrm{D} \nu \mathrm{M}$. To demonstrate this point, Ref. [7] compared the detected neutrino light-curves obtained for 1D "nonexploding" and 2D "exploding" simulations of the same progenitor. The analysis in [7] therefore closely overlaps with our current work. There are, however, important differences. First, Ref. [7] altogether ignored pES in JUNO. As we discuss (see also $[46,52,53,62]$ ), pES is actually the second most important class of events (after IBD) in JUNO, assuming a realistic lower energy threshold, and it plays a key role in the current work. Second, while Ref. [7] presented qualitative comparisons between the expected neutrino signals for "exploding" vs "nonexploding" simulations, it focused solely on integrated total event rates and did not analyze in detail the prospects to actually discriminate between the two cases in a detection. Using total event rates, this discrimination would be challenging. ${ }^{6}$ One of our main results in this work is that flavor information,

\footnotetext{
${ }^{6}$ Compare, for example, the JUNO event rates presented in the left ("nonexploding") and right ("exploding") panels in Fig. 11 in [7], and recall that in a detection, the actual structure of the progenitor would be unknown.
} 

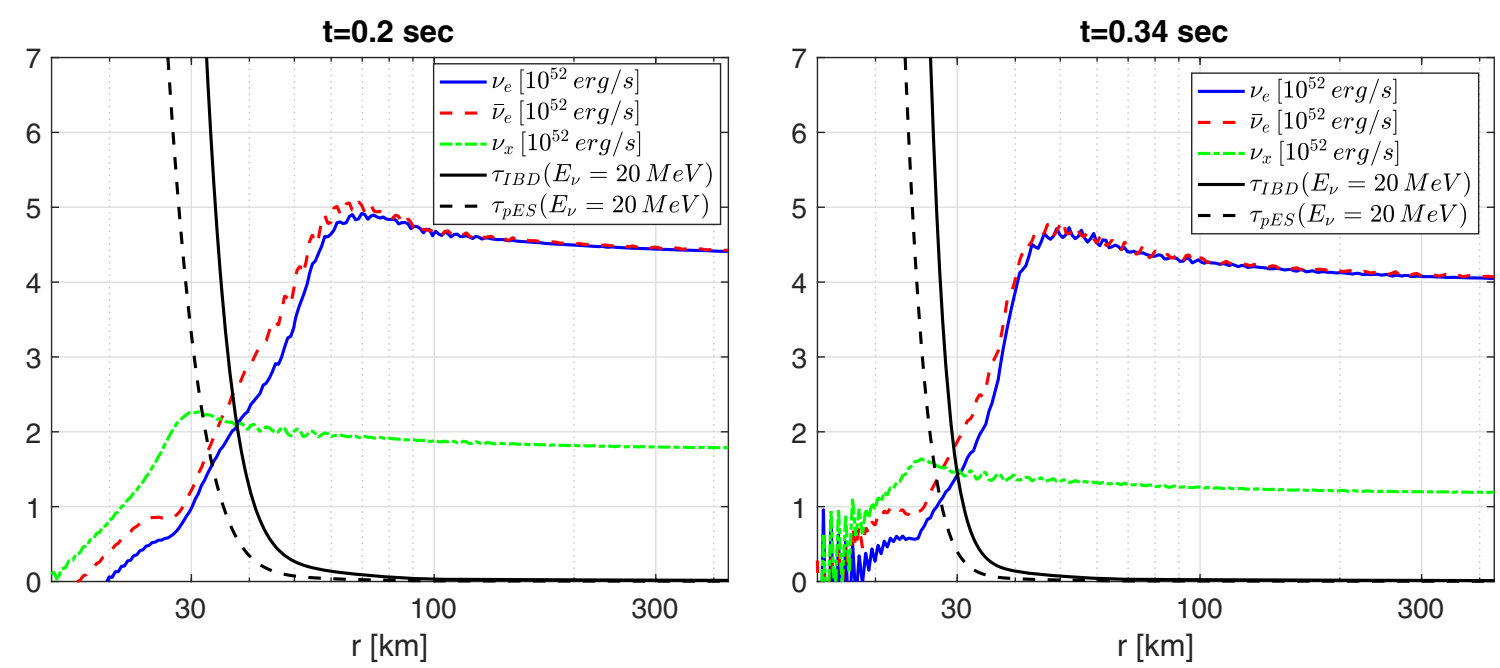

FIG. 2. Snapshots of the radial profiles of the neutrino luminosity per flavor (solid blue, dashed red, dot-dashed green referring to $\nu_{e}$, $\bar{\nu}_{e}, \nu_{x}$, respectively), during the accretion phase, calculated with GR1D. Solid (dashed) black line shows the optical depth to scattering for IBD (pES) for a neutrino with energy $E_{\nu}=20 \mathrm{MeV}$. Left: $t=0.2 \mathrm{sec}$ post-bounce. Right: $t=0.34$ sec postbounce.

specifically the $\mathrm{pES} / \mathrm{IBD}$ event rate ratio, provides a robust test of a continued accretion phase.

This paper is structured as follows. In Sec. II we demonstrate the basic physical features of the accretiondominated neutrino luminosity, expected to characterise the neutrino light-curve in CITE and also in the early stages of the CCSN in the D $\nu \mathrm{M}$. We use numerical simulations to illustrate the discussion. For completeness, in Appendix A we review analogous results from numerical simulations conducted by different groups and using different codes.

In Sec. III we discuss channels for CCSN neutrino detection in a scintillation detector, focusing on JUNO. We highlight the IBD and pES channels, which are dominant in terms of event statistics, and explain our analysis strategy including cuts in the deposited energy spectrum. The importance of achieving a low quenched proton recoil energy threshold is explained. Some cross section formulas are collected in Appendix B.

In Sec. IV we describe our treatment of neutrino flavor conversion in the CCSN, relating flavor-specific neutrino source luminosities to the luminosities at Earth. In the case of CITE, neutrino propagation proceeds in the deep adiabatic regime throughout the first few seconds after core-collapse. We explain this result in Appendix C.

In Sec. $\mathrm{V}$ we analyze results from numerical simulations. Our main goal in this section is to explore (i) the theoretical uncertainty arising from different progenitor stars and EoS, and (ii) realistic statistical uncertainties for a galactic CCSN. We also study the impact of uncertainties in neutrino oscillation parameters.

In Sec. VI we summarize our results.

The numerical simulations we use in the paper are 1D. In Appendix D we show that core rotation-that is required to some extent, at least in the CITE scenario-is expected to make only a small perturbation over the 1D results during the first few seconds of the neutrino burst.

\section{II. $\bar{\nu}_{e} / \nu_{x}$ RATIO DURING THE ACCRETION PHASE}

Our goal in this section is to clarify the origin of the accretion luminosity, that leads to excess $\nu_{e}, \bar{\nu}_{e}$ compared to the $\mathrm{x}$-flavors. In Fig. 2 we show radial profiles of the neutrino luminosity per flavor at fixed time, for two postbounce times $t=0.2,0.34 \mathrm{sec}$ (left and right panels, respectively) during the accretion phase, calculated with the (nonexploding) open-source general-relativistic hydrodynamics 1D code GR1D [69,70] for a $15 M_{\odot}$ progenitor star, ${ }^{7}$ with the SLy4 EoS [71,72]. Black solid (dashed) lines show the optical depth to IBD (pES), the first being the key quantity for $\bar{\nu}_{e}$ and the second for $\nu_{x}$. Both are computed for neutrino energy $E_{\nu}=20 \mathrm{MeV}$.

The $\nu_{e}, \bar{\nu}_{e}$ luminosities in Fig. 2 are dominated by nucleon conversion, $p+e^{-} \rightarrow n+\nu_{e}$ and $n+e^{+} \rightarrow p+\bar{\nu}_{e}$, and $e^{-} e^{+}$annihilation taking place in the region below the accretion shock and above the neutrinosphere. $L_{\bar{\nu}_{e}}$ and $L_{\nu_{e}}$ continue to build up in the optically thin region $\left(\tau_{p \mathrm{ES}}\right.$, $\tau_{\mathrm{IBD}}<1$ ), all the way to the location of the accretion shock, at which point the luminosity saturates. The contribution to the luminosity coming from the optically thin region, is what we refer to as accretion luminosity. In contrast, $\mathrm{x}$-flavor emission saturates on smaller radii near the $\mathrm{x}$-flavor neutrinosphere $\left(\tau_{p \mathrm{ES}} \approx 1\right)$.

The ratio $f_{\bar{e}}=L_{\bar{\nu}_{e}} / L_{\nu_{x}}$, computed for both panels of Fig. 2, is shown in Fig. 3 as a function of distance from the

\footnotetext{
${ }^{7}$ Our progenitor profiles are taken from the nonrotating, solar metallicity sample of [40] (see https://2sn.org/stellarevolution/ data.shtml). Masses refer to zero-age main-sequence.
} 


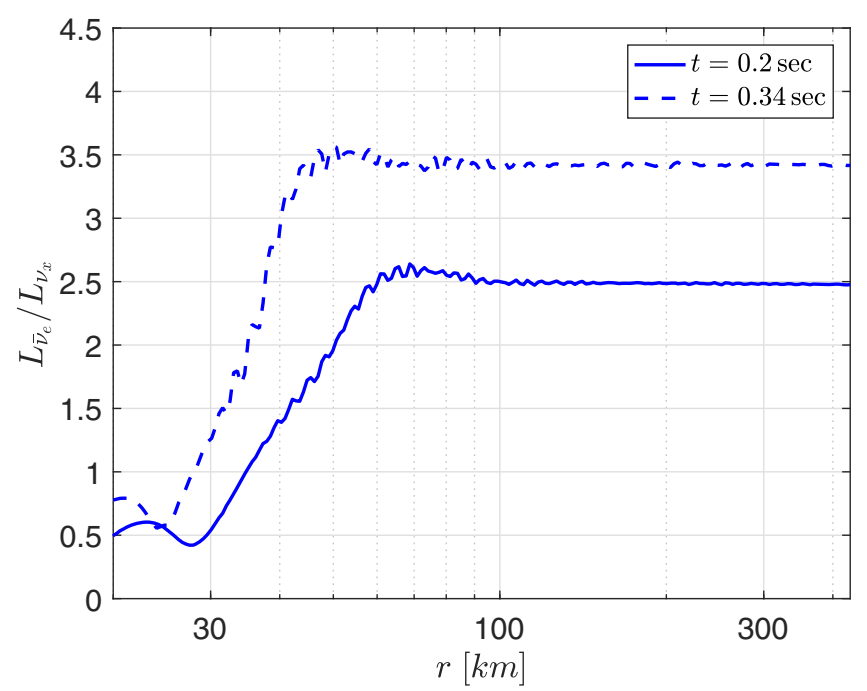

FIG. 3. Snapshots of the radial profile of $f_{e}=L_{\bar{\nu}_{e}} / L_{\nu_{x}}$ during the accretion phase, calculated with GR1D. Smooth (dashed) lines refer to snapshots at $t=0.2 \mathrm{sec}(t=0.34 \mathrm{sec})$ postbounce.

centre of the star. In Fig. 4 we show the per-flavor neutrino luminosity crossing through $r=400 \mathrm{~km}$ as a function of time, for the same simulation. Note that Fig. 4 does not include the effect of neutrino oscillations. The effect of oscillations will be accounted for below.

The details of the neutrino burst vary between progenitor stars and depend also on the assumed EoS. However, e-flavor dominance during the accretion phase is a generic phenomenon. We illustrate this point in Fig. 5, where we show results calculated for a set of five simulations: (i) SLy4 EoS, $15 M_{\odot}$ star (thick black, the same simulation used in Figs. 2-4), (ii) SLy4 EoS, $30 M_{\odot}$ (dashed magenta), (iii) LS220 [72,73], $15 M_{\odot}$ (blue) (iv) LS220, $30 M_{\odot}$ (dashed red), (v) KDE0v1 EoS [74], $20 M_{\odot}$ (dotted green). On the left panel we show the $\bar{\nu}_{e}$ luminosity, and on the right we show the ratio $L_{\bar{\nu}_{e}} / L_{\nu_{x}}$, both calculated at the source $(r=400 \mathrm{~km})$.

In Appendix A we review two additional examples from the literature [7,75], using different simulation codes. Reference [75] compared the neutrino light-curves of failed explosions, to the light-curves of simulations in which an explosion was set-off by hand. Similarly, Ref. [7] compared 1D "failed" and 2D "successful" explosions in simulations of the same progenitor stars. ${ }^{8}$ The failed explosions in $[7,75]$ give similar results to the GR1D simulations that we explore here: the different calculations agree on $f_{\bar{e}}>2$ during the accretion phase.

The successful explosions in [7,75] serve to illustrate the $\mathrm{D} \nu \mathrm{M}$ scenario. We cannot test this scenario directly with GR1D (without setting-off artificial explosions). Instead, we will consider a simple PNS cooling scenario to crudely

\footnotetext{
${ }^{8}$ Seed perturbations and numerical noise were used to initiate turbulence in the 2D models analyzed in [7]; see Sec. V there.
}

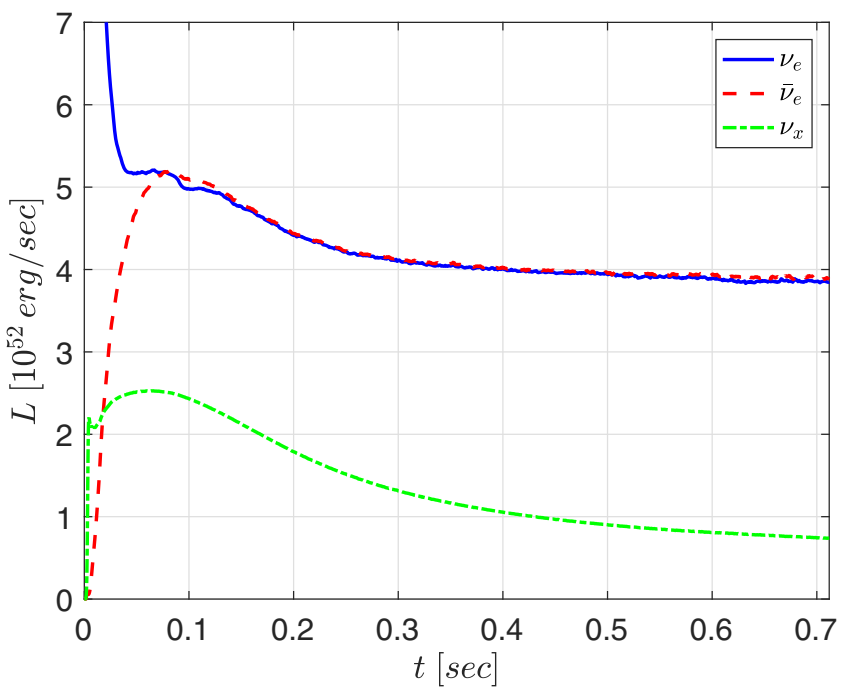

FIG. 4. Time-dependent neutrino luminosity crossing through $r=400 \mathrm{~km}$ during the accretion phase, calculated with GR1D. In this plot, we do not include neutrino oscillations, the effect of which will be accounted for later. $t$ is time post-bounce.

approximate the $\mathrm{D} \nu \mathrm{M}$. We define the PNS cooling limit by setting the e-flavor spectra equal to the x-flavor spectra. This is a reasonable approximation, e.g., to the results of [75]. However, comparing to [7], we note that residual accretion leads to some enhanced e-flavor emission, with $f_{e} \sim 1.5$. This demonstrates the limitations of the simple PNS cooling prescription, implying that our analysis cannot test the $\mathrm{D} \nu \mathrm{M}$ at the same level of robustness by which it can test CITE.

\section{NEUTRINO DETECTION CHANNELS IN A SCINTILLATION DETECTOR}

The JUNO detector [46], ${ }^{9}$ currently under construction, will contain 20 kton of linear alkylbenzene (LAB, composed of $\left.\mathrm{C}_{6} \mathrm{H}_{5} \mathrm{C}_{n} \mathrm{H}_{2 n+1}\right)$. In calculations, we round off the available number of target protons to $N_{p}=1.5 \times 10^{33}$. For $n$ in the range 10-13, the available number of target electrons is $N_{e}=(4.63 \pm 0.065) \times N_{p}$. In what follows, we describe the neutrino detection channels that are relevant for the CCSN analysis $[62,76]$.

The detection channels available in JUNO include IBD, pES, eES, and neutrino-nucleus $(\nu \mathrm{N})$ charged- and neutralcurrent scattering. We mainly focus on the IBD and $\mathrm{pES}$ channels, which are expected to dominate the event rate and which contain the flavor information we are after in this study. The different channels are characterized by distinct deposited energy spectra. This, combined with additional event-by-event information (such as the gamma-rays due to

\footnotetext{
${ }^{9}$ Our analysis applies equally well to the proposed 50 kton LENA [47] detector.
} 

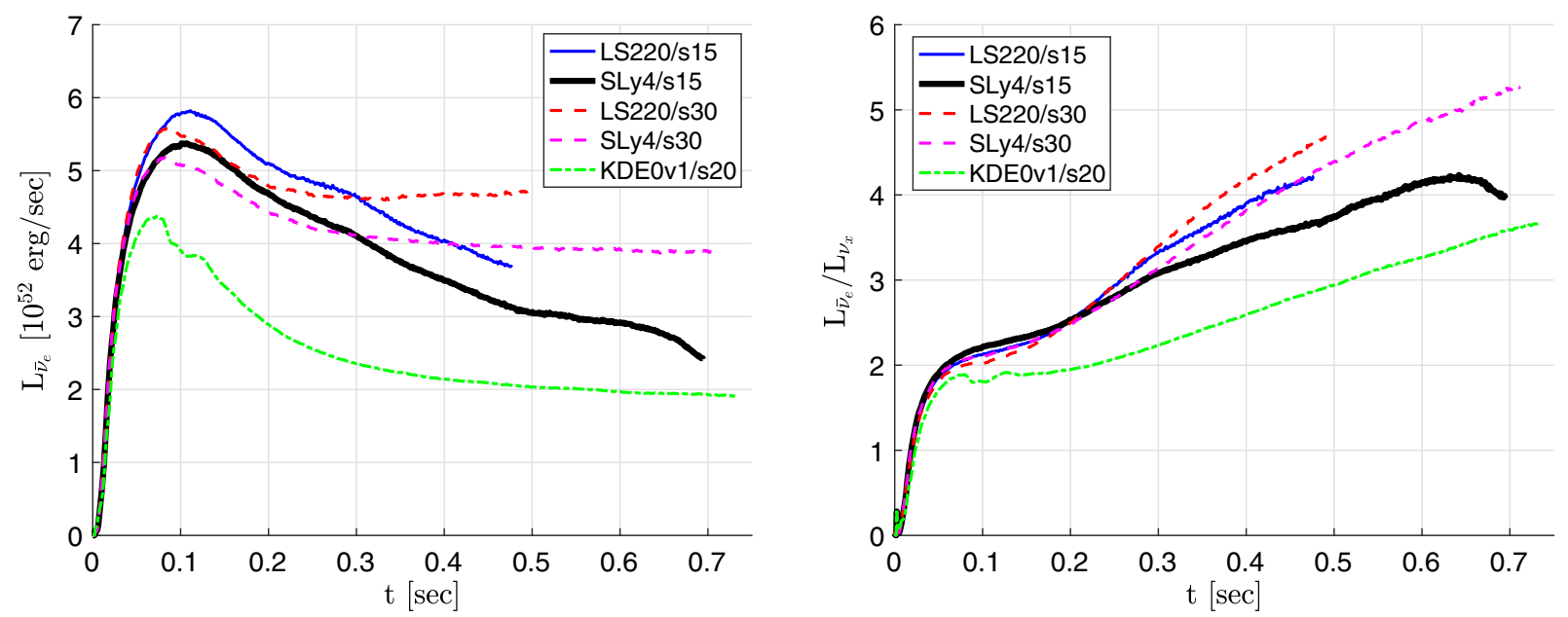

FIG. 5. Time-dependent neutrino luminosity at the source $(r=400 \mathrm{~km}) . t$ is time post-bounce. Left: $L_{\bar{\nu}_{e}}$. Right: The ratio $L_{\bar{\nu}_{e}} / L_{\nu_{x}}$. We do not include neutrino oscillations, the effect of which will be accounted for later. Results are shown for the five simulations described in the text.

neutron capture and $e^{+}$annihilation for IBD), allows an analysis in JUNO to effectively tag the different channels.

Considering background contamination, this would not affect the result for IBD [46] but could be significant for low-threshold pES [52,53]. Given that a detailed spectral background measurement will become available with the completion of JUNO, we account for backgrounds effectively by considering different values for the lower threshold deposited energy in proton recoil, discussed in Sec. III B. As we will show, the lower the threshold, the better are the capabilities of the flavor analysis.

\section{A. IBD $\left(\bar{\nu}_{e}+p \rightarrow e^{+}+n\right)$}

We adopt the approximation used in [34], exploiting the weak dependence of the cross section on the scattering angle. The incoming neutrino energy $E_{\nu}$ is wellreconstructed by the measured positron energy $E_{e}$, via ${ }^{10}$

$$
E_{\nu} \approx \frac{E_{e}+Q}{1-E_{e} / m_{p}}, \quad Q=m_{n}-m_{p}=1.293 \mathrm{MeV} .
$$

The rate of detected events is then

$$
\dot{N}_{\mathrm{IBD}}=N_{p} \int_{E_{e}^{\min }}^{E_{e}^{\max }} d E_{e} \Phi_{\bar{\nu}_{e}}^{\oplus}\left(E_{\nu}, t\right) \sigma_{\mathrm{IBD}}\left(E_{\nu}\right) J\left(E_{\nu}\right) .
$$

Here $\Phi_{\nu_{\alpha}}^{\oplus}$ is the neutrino flux at Earth, we use Eq. (3) to compute $E_{\nu}\left(E_{e}\right)$ in the integral, and the Jacobian is $J\left(E_{\nu}\right)=$ $\left(1+E_{\nu} / m_{p}\right)^{2} /\left(1+Q / m_{p}\right)$. The minimum detectable neutrino energy is $Q+m_{e}$, for which the positron is emitted at $E_{e}=m_{e}$. The details of the choice of $E_{e}^{\min }$ are not very

\footnotetext{
${ }^{10}$ The exact result is $E_{\nu}=\left(E_{e}+\delta\right) /\left[1-\left(E_{e}-p_{e} \cos \theta\right) / m_{p}\right]$, where $\delta \equiv\left(m_{n}^{2}-m_{p}^{2}-m_{e}^{2}\right) / 2 m_{p}, p_{e}$ is the positron momentum and $\theta$ is the scattering angle of the positron.
}

important; in practice, we choose $E_{e}^{\min }=2 \mathrm{MeV}$. We take $E_{e}^{\max }=100 \mathrm{MeV}$, noting that the actual neutrino spectrum dies off exponentially above a few tens of MeV. The explicit form of $\sigma_{\mathrm{IBD}}$ is recalled in Appendix B. For the range of neutrino energies we are interested in, $\sigma_{\mathrm{IBD}}$ is known with less than $1 \%$ uncertainty. Finally, note that the IBD events can be tagged by, e.g., neutron capture, with tagging efficiency estimated at about $90 \%$ [62].

\section{B. $\mathrm{pES}\left(\nu_{\alpha}+p \rightarrow \nu_{\alpha}+p\right)$}

In a neutrino-proton elastic scattering (pES) [52], the proton receives some recoil energy $T$. However, due to the quenching process of the scintillator, the observed energy of the proton, $T^{\prime}$, is smaller. The relation $T^{\prime}(T)$ is dictated for each detector by the solvent, using the semiempirical Birk's law [77]. In what follows, we use $T^{\prime}(T)$ taken from [46], noting that accurate measurements of this relation [78] can be conducted in situ once JUNO begins operation.

The rate of detected events per unit reconstructed recoil energy is [53]

$\frac{d \dot{N}_{\mathrm{pES}}}{d T^{\prime}}=\frac{N_{p}}{\left(d T^{\prime} / d T\right)} \int_{E_{\nu}^{\min }(T)}^{\infty} d E_{\nu} \sum_{\nu_{\alpha}, \bar{\nu}_{\alpha}} \Phi_{\nu_{\alpha}}^{\oplus}\left(E_{\nu}\right) \frac{d \sigma_{\mathrm{pES}}\left(E_{\nu}, T\right)}{d T}$.

The sum includes all flavors of neutrinos and antineutrinos. The differential cross section $\frac{d \sigma_{\mathrm{PES}}\left(E_{\nu}, T\right)}{d T}$, recalled in Appendix B, is independent of incoming neutrino flavor. Therefore, for unitary three-flavor neutrino propagation, the pES channel depends only on the total all-flavor neutrino flux at the source and is independent of the details of neutrino oscillations [52]. 

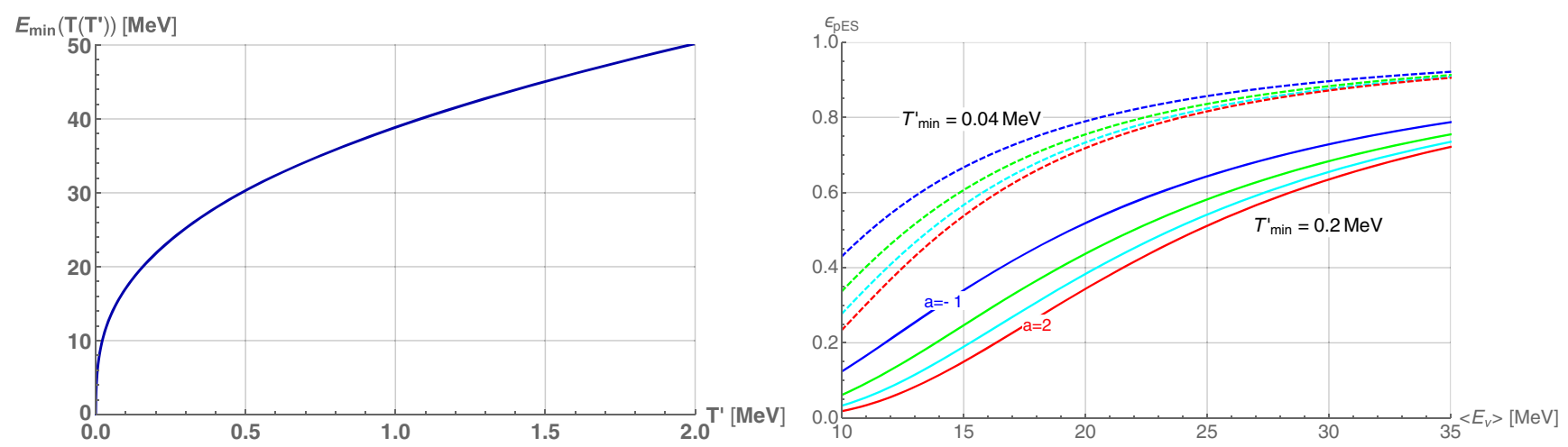

FIG. 6. Left: The minimal neutrino energy required to induce a reaction with observable (quenched) energy $T^{\prime}$. Right: pES efficiency $\epsilon_{\mathrm{pES}}$, calculated for the pinched Fermi-Dirac spectrum as function of the mean neutrino energy $\left\langle E_{\nu}\right\rangle$ for recoil energy threshold $T_{\min }^{\prime}=0.2$ (solid) and $T_{\min }^{\prime}=0.04$ (dashed). The four lines (for each value of $T_{\min }^{\prime}$ ) correspond to $a=-1,0,1,2$, from top (blue) to bottom (red).

The minimum detectable neutrino energy is ${ }^{11}$

$$
E_{\nu}^{\min }(T)=\sqrt{\left(1+\frac{T}{2 m_{p}}\right) \frac{m_{p} T}{2}}+\frac{T}{2}
$$

The event rate is then

$$
\dot{N}_{\mathrm{pES}}=N_{p} \int_{T_{\min }}^{T_{\max }} d T \int_{E_{\nu}^{\min }(T)}^{\infty} d E_{\nu} \sum_{\nu_{\alpha}, \bar{\nu}_{\alpha}} \Phi_{\nu_{\alpha}}^{\oplus}\left(E_{\nu}\right) \frac{d \sigma_{\mathrm{pES}}\left(E_{\nu}, T\right)}{d T} .
$$

The integration over proton recoil energy runs between $T_{\min }$ to $T_{\max }$. These recoil energy limits are derived from quenched (observed) reconstructed energy limits, $T_{\min }^{\prime}$ and $T_{\max }^{\prime}$, using the relation $T^{\prime}(T)$. In the current work we set the upper limit $T_{\max }^{\prime}=2 \mathrm{MeV}$, for which the corresponding minimal incoming neutrino energy is $E_{\nu}^{\min }\left(T\left(T_{\max }^{\prime}\right)\right) \approx 50 \mathrm{MeV}$. We set this $T_{\max }^{\prime}$ cut in order to decrease the contamination of eES events in the $\mathrm{pES}$ sample. As long as $T_{\max }^{\prime} \sim 2 \mathrm{MeV}$, the details of the choice of $T_{\max }^{\prime}$ are not very important for our analysis, although changing the $T_{\max }^{\prime}$ cut around $1-3 \mathrm{MeV}$ would make a small (controllable) numerical change to the expected value of our flavor observable.

The lower threshold $T_{\min }^{\prime}$ is more important and requires discussion. The left panel of Fig. 6 shows the minimal neutrino energy required to induce a reaction with observable quenched energy $T^{\prime}$. CCSN neutrinos carry characteristic energies of between $\sim 10$ to a few 10's of MeV, so the value of the quenched energy threshold $T_{\min }^{\prime}$ can have a significant effect on the detection efficiency. To estimate

\footnotetext{
${ }^{11}$ Reference [53] used the approximation $E_{\nu}^{\min }(T) \approx \sqrt{m_{p} T / 2}$. We find that this approximation leads to a $\sim 15 \%$ error in the total event rate, because most of the contribution to the integral is typically close to the lower integration limit in a CCSN scenario.
}

the effect, consider a pinched Fermi-Dirac representation of the neutrino flux at Earth for a CCSN at distance $D_{\mathrm{SN}}$,

$$
\Phi_{\nu, \mathrm{FD}}^{\oplus}=\frac{L}{4 \pi D_{\mathrm{SN}}^{2} c_{L}(a) T^{2}} \frac{\left(E_{\nu} / T\right)^{2+a}}{\exp \left(E_{\nu} / T\right)+1} .
$$

This spectrum is parametrized by three numbers: the pinch index $a$, the temperature $T$, and the source luminosity $L$. We define $c_{L}(a)=\left(1-2^{-3-a}\right) \Gamma(4+a) \zeta(4+a)$, and note that the mean neutrino energy $\left\langle E_{\nu}\right\rangle$ for this spectrum is given by $\left\langle E_{\nu}\right\rangle=c_{T}(a) T_{\nu}$, where $c_{T}(a)=$ $\frac{\left(2^{3+a}-1\right) \Gamma(4+a) \zeta(4+a)}{2\left(2^{2+a}-1\right) \Gamma(3+a) \zeta(3+a)}$. Using Eq. (8), and neglecting the upper threshold $T_{\max }^{\prime}$, we can calculate the pES efficiency as the ratio between the number of $\mathrm{pES}$ events detected with finite $T_{\min }^{\prime}$ threshold to the number of events that would be detected with $T_{\min }^{\prime} \rightarrow 0$,

$\epsilon_{\mathrm{pES}}\left(T_{\min }^{\prime}\right) \approx \frac{\int_{T_{\min }}^{\infty} d T \int_{E_{\nu}^{\min }(T)}^{\infty} d E_{\nu} \Phi_{\nu, \mathrm{FD}}^{\oplus}\left(E_{\nu}\right) \frac{d \sigma_{\mathrm{pES}}\left(E_{\nu}, T\right)}{d T}}{\int_{0}^{\infty} d T \int_{E_{\nu}^{\min }(T)}^{\infty} d E_{\nu} \Phi_{\nu, \mathrm{FD}}^{\oplus}\left(E_{\nu}\right) \frac{d \sigma_{\mathrm{pES}}\left(E_{\nu}, T\right)}{d T}}$.

In modeling JUNO we will show results using two values of the lower quenched energy threshold, $T_{\min }^{\prime}=0.2 \mathrm{MeV}$ (the main value used in [46]), along with an optimistic $T_{\min }^{\prime}=0.04 \mathrm{MeV}$. The resulting $\epsilon_{\mathrm{pES}}$ is shown in the right panel of Fig. 6.

We emphasize that Eq. (9) and the spectral model of Eq. (8) are not used in our numerical calculations, in which we take neutrino spectra directly from the simulations and use the full cross section expressions to calculate event rates. Nevertheless, in a realistic CCSN, the flux of neutrinos of flavor $\nu_{\alpha}$ is reasonably well described by Eq. (8) with flavor-dependent parameters, $(a, T, L) \rightarrow$ $\left(a_{\nu_{\alpha}}, T_{\nu_{\alpha}}, L_{\nu_{\alpha}}\right)$, and with a weight corresponding to the oscillation probability: $\Phi_{\nu_{\alpha}}^{\oplus} \approx \sum_{\nu_{\beta}} P_{\alpha \beta} \Phi_{\nu_{\beta}, \mathrm{FD}}^{\oplus}$. Thus, the right panel of Fig. 6 gives a useful illustration of the impact of $T_{\min }^{\prime}$. 
Finally, it is important to note that current uncertainty on the strangeness content of the proton [79-81] induces a $\sim 20 \%$ uncertainty on $\sigma_{\mathrm{pES}}$. However, there is a proposal to reduce this uncertainty by an order of magnitude [51]. We will assume here that the uncertainty on $\sigma_{\mathrm{pES}}$ can indeed be reduced to the few percent level.

\section{Additional channels: eES and neutrino-nucleus scattering}

\section{1. $e E S\left(\nu_{\alpha}+e \rightarrow \nu_{\alpha}+e\right)$}

The cross section for neutrino-electron elastic scattering (eES) is summarized in Appendix B. The cross section depends on the incoming neutrino flavor, with $\sigma_{\nu_{e} e} / \sigma_{\bar{\nu}_{e} e} \approx$ 2.38, $\sigma_{\nu_{e} e} / \sigma_{\nu_{x} e} \approx 5.81, \sigma_{\nu_{e} e} / \sigma_{\bar{\nu}_{x} e} \approx 7.15$. The eES cross section is smaller than the pES cross section by a factor of $\approx 22,43,65$ for $E_{\nu}=10,20,30 \mathrm{MeV}$, respectively, where for this comparison we considered incoming $\nu_{e}$. Therefore, despite the target enhancement factor of $N_{e} / N_{p} \approx 4.6$, the total eES event rate is smaller than the total $\mathrm{pES}$ event rate by a factor of $\sim 10$, provided that the analysis can achieve $T_{\min }^{\prime} \lesssim 0.2 \mathrm{MeV}$ in pES detection.

The kinematics of eES is the same as that in $\mathrm{pES}$, up to replacing $m_{p} \rightarrow m_{e}$. The electron recoil energy is $T_{e} \equiv$ $E_{e}-m_{e}=\frac{1-\cos \theta}{1+\left(1-\cos \theta \frac{E_{\nu}}{m_{e}}\right.} \frac{E_{\nu}^{2}}{m_{e}}$, where $\theta$ is the scattering angle of the neutrino. For a typical CCSN neutrino, $E_{\nu} \gg m_{e}$ and thus $T_{e} \sim E_{\nu}$. As a result, the deposited energy spectra of eES and pES are separated, the former peaking at higher deposited energy than the latter. With our upper cut of $T_{\max }^{\prime}=2 \mathrm{MeV}$, the eES contribution in the elastic scattering sample will be at the percent level of that from pES.

\section{Neutrino-nucleus scattering}

The neutrino-nucleus charged current (CC) $\nu_{e}{ }^{12} \mathrm{C} \rightarrow$ $e^{-12} \mathrm{~N}, \bar{\nu}_{e}{ }^{12} \mathrm{C} \rightarrow e^{+12} \mathrm{~B}, \nu \nu_{e}{ }^{13} \mathrm{C} \rightarrow e^{-13} \mathrm{~N}$ and neutral current (NC) $\nu^{12} \mathrm{C} \rightarrow \nu^{12} \mathrm{C}^{*}, \nu^{13} \mathrm{C} \rightarrow \nu^{13} \mathrm{C}^{*}$ interactions would also contribute some events in JUNO $[7,46,55,62,76]$ (see also $[60,82]$ for $\nu \mathrm{O}$ scattering). The recoil energy of the nucleus is too small to be measured directly. The expected number of NC events is below about $10 \%$ of the IBD sample [62], and the channel can be effectively identified via the monochromatic deexitation gamma rays. The expected number of CC events is comparable to $\mathrm{NC}$ and eES. The deposited energy spectra due to the CC final state $e^{-}$or $e^{+}$are peaked in approximately the same range as the IBD events, and die off faster than eES towards low deposited energy [62]. With a cut of $T_{\max }^{\prime}=2 \mathrm{MeV}$, the contamination of $\mathrm{CC}$ events to the $\mathrm{pES}$ channel is negligible.

In summary, neutrino-nucleus events are statistically subdominant, and with simple event tagging and analysis cuts, would not contaminate the IBD and pES samples. In our analysis we therefore do not consider these channels further, noting only that their detection would add more information to a CCSN signal analysis beyond what we offer in this work $[46,55,62,76]$.

\section{NEUTRINO OSCILLATIONS}

Neutrino flavor conversion in the supernova is computed using the Mikheyev-Smirnov-Wolfenstein formalism $[83,84]$ (if self-induced oscillations are not important; however, see discussion below). Focusing on the oscillations of antineutrinos, important for the IBD channel, the survival probability of $\bar{\nu}_{e}$ is given by [85]

$$
\begin{aligned}
P_{\bar{e} \bar{e}} & = \begin{cases}\left|U_{e 1}\right|^{2}\left(1-P_{\bar{L}}\right)+\left|U_{e 2}\right|^{2} P_{\bar{L}}, & \mathrm{NH}, \\
\left|U_{e 1}\right|^{2} P_{\bar{H}}\left(1-P_{\bar{L}}\right)+\left|U_{e 2}\right|^{2} P_{\bar{H}} P_{\bar{L}}+\left|U_{e 3}\right|^{2}\left(1-P_{\bar{H}}\right), & \mathrm{IH},\end{cases} \\
& \approx \begin{cases}0.68-0.38 P_{\bar{L}}, & \mathrm{NH}, \\
0.02+\left(0.66-0.38 P_{\bar{L}}\right) P_{\bar{H}}, & \mathrm{IH} .\end{cases}
\end{aligned}
$$

$P_{\bar{H}}$ and $P_{\bar{L}}$ are the level crossing probabilities between propagation eigenstates for the so-called $H$ - and $L$ - (antineutrino) subsystems, respectively. The adiabatic limit amounts to setting $P_{\bar{L}}=P_{\bar{H}}=0$. NH and IH refer to normal mass hierarchy and inverted mass hierarchy, respectively.

Even for prolonged accretion, relevant for the CITE scenario, we demonstrate in Appendix $\mathrm{C}$ that the adiabatic limit is justified at least in the first few seconds $\left(t_{\mathrm{PB}} \lesssim 5 \mathrm{sec}\right)$ of the neutrino burst. For the $\mathrm{D} \nu \mathrm{M}$, once explosion happens the situation may be more complicated because some of the matter overburden is swept away such that level-crossing resonance regions may be traversed in the nonadiabatic regime [37]. Nevertheless, the adiabatic limit is still a useful benchmark, used extensively in analyses of SN1987A [32-35] and in the discussion of a future galactic CCSN [8,60,61,63,85-88]. Formulating clear predictions for the neutrino signal in this limit would allow to identify and interpret possible deviations in real data. We therefore adopt this limit in most of our current work. Assuming that the $\mathrm{x}$-flavor spectra are equal at the source, the $\bar{\nu}_{e}$ flux at earth is related to the fluxes at the source via (note that in our notation, $\Phi_{\bar{\nu}_{x}}$ is the average flux per species of $\bar{\nu}_{\mu}, \bar{\nu}_{\tau}$ )

$$
\Phi_{\bar{\nu}_{e}}^{\oplus}=P_{\bar{e} \bar{e}} \Phi_{\bar{\nu}_{e}}+\left(1-P_{\bar{e} \bar{e}}\right) \Phi_{\bar{\nu}_{x}} .
$$



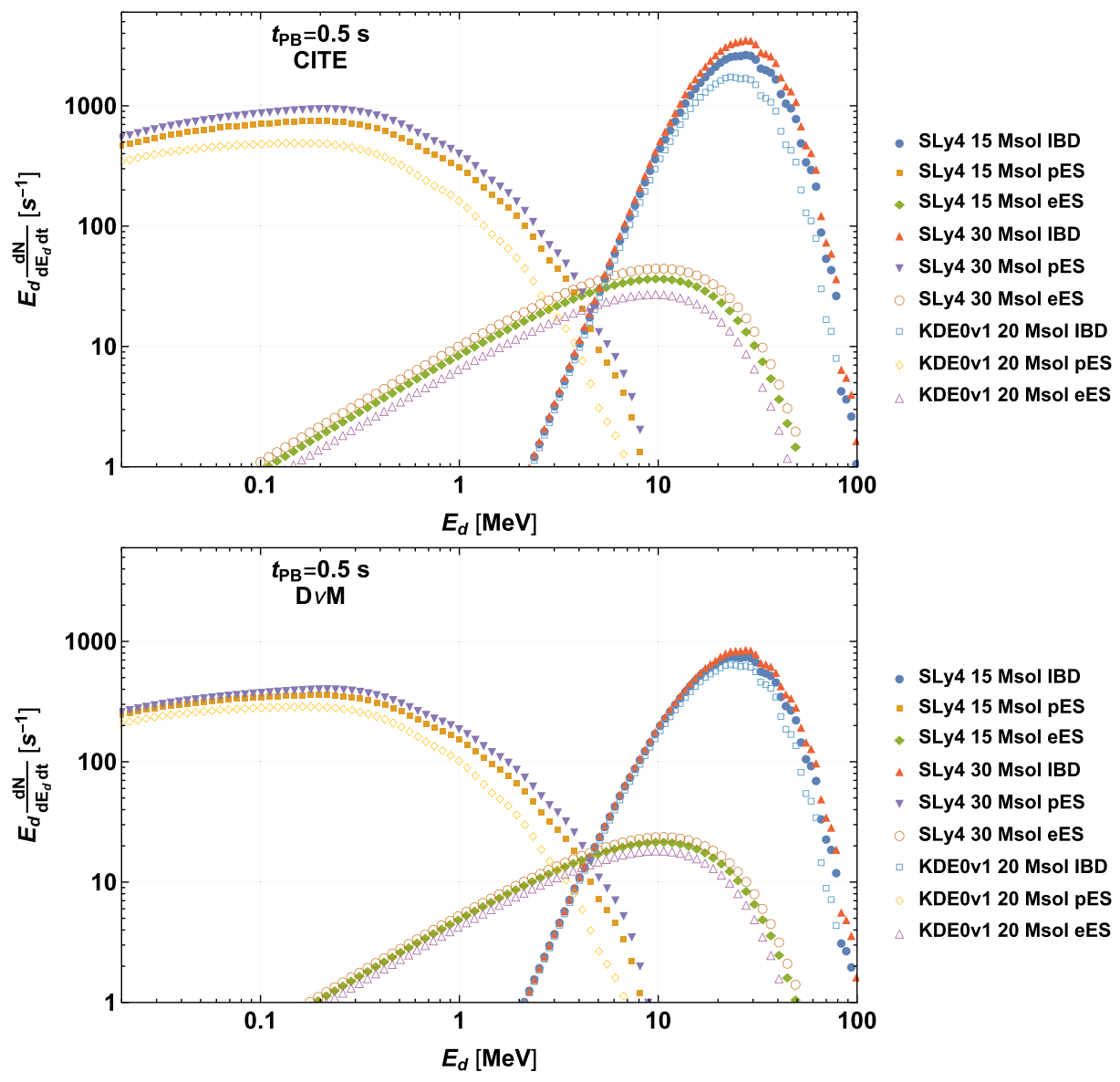

FIG. 7. Event rate vs deposited energy at JUNO. The broad bump peaking around $E_{d} \approx 0.3 \mathrm{MeV}$ is due to pES. The dominant, narrower bump peaking around $E_{d} \approx 30 \mathrm{MeV}$ is due to IBD. The subdominant bump (lower than the peak pES rate by a factor of $\sim 100$ ) is due to eES. We plot a snapshot of the predicted detection spectrum at $t_{\mathrm{PB}}=0.5 \mathrm{sec}$, for adiabatic matter flavor conversion assuming normal hierarchy. The CCSN simulations corresponding to the different markers are explained in the text. Top: CITE. Bottom: PNS cooling, representing a simple limit of the $\mathrm{D} \nu \mathrm{M}$.

In the numerical expressions above and in our main analysis, we use best-fit values for the oscillation parameters, taken from [89,90].

Self-induced neutrino oscillations may significantly affect the adiabatic Mikheyev-Smirnov-Wolfenstein prediction [91-99]. The problem, however, is not settled yet. In particular, during the accretion phase the large matter density may inhibit the self-induced oscillations effect $[48,50,100-109]$. Since the jury is still out on the outcome and importance of self-induced oscillations, we study the impact of different oscillation probabilities in Sec. VA, spanning the range $P_{\bar{e} \bar{e}}=[0,1]$. This also serves to exhibit the sensitivity of our main results, using Eq. (10), to measurement uncertainties in the oscillation parameters.

Finally, note that neutrino propagation is also slightly affected by matter in the Earth $[85,110]$. The effect depends on directionality, which would be known accurately for a future galactic CCSN event allowing corrections to our formalism to be implemented if needed. We therefore omit Earth matter effects in this work.

\section{NUMERICAL SIMULATIONS OF CCSN DETECTION IN JUNO}

With the calculations of Secs. III and IV, we can use numerical simulations of core-collapse to predict $\mathrm{pES}$ and IBD event rates at JUNO. We will find the simple counting observable $R$, introduced in Eq. (2), particularly useful. In what follows, after some discussion of the spectral shape of the signal, we analyze $R$ for simulated bursts. We then carry out a likelihood analysis of mock data.

We first study spectral information. Figure 7 shows a temporal snapshot, taken at $t_{\mathrm{PB}}=0.5 \mathrm{sec}$, of the deposited energy spectra in JUNO. To produce this plot, we assume a CCSN at $10 \mathrm{kpc}$ [36] with adiabatic matter-induced flavor conversion and normal hierarchy. We show the results of 3 different simulation runs, computed with GR1D. The first two runs use the SLy4 EoS [71] for $15 M_{\odot}$ and $30 M_{\odot}$ stars. The third run uses the KDE0v1 EoS [74] and a $20 M_{\odot}$ star. In the top panel we show the deposited energy spectra obtained directly from the simulations. These 
results correspond to the CITE scenario. In the bottom panel we show an estimate of what the deposited energy spectra are expected to look like in the PNS cooling limit of the D $\nu \mathrm{M}$. To obtain this estimate, we set the $\nu_{e}$ and $\bar{\nu}_{e}$ luminosities at the source equal to the $\nu_{x}$ luminosity.

The event spectra in the top panel of Fig. 7 (CITE) are higher by a factor of 2-3 compared to the rates in the bottom panel (PNS cooling). This overall enhancement is interesting for estimates of the diffuse supernova neutrino background (DSNB) [111]. However, it would only give a partial hint toward diagnosing the explosion mechanism underlying a single galactic explosion, because it is degenerate with details of the stellar profile and the nuclear EoS. Rather than the total luminosity, a key difference between the top and bottom panels is the relative height of the $\mathrm{pES}$ and IBD peaks. This information is captured by $R$.

\section{A. Simple counting observable: $R=p E S / I B D$.}

In this section we study $R$ as a diagnostic of the neutrino emission process. We start in Sec. VA 1 by analyzing numerical simulations, and discuss the sensitivity of our results to modeling uncertainties in Sec. VA2.

\section{Analysis of core-collapse simulations}

The top panel of Fig. 8 shows the observable $R$ vs postbounce time $t_{\mathrm{PB}}$, binned in $0.1 \mathrm{sec}$ time segments, calculated for a galactic CCSN at $10 \mathrm{kpc}$ and measured by JUNO with a deposited energy threshold $T_{\min }^{\prime}=0.2 \mathrm{MeV}$. Thick lines (all below $R=0.5$ ) correspond to CITE. Thin lines (all above $R=0.5$ ) correspond to the PNS cooling limit of the $\mathrm{D} \nu \mathrm{M}$. We show results from GR1D simulations of $15 M_{\odot}, 20 M_{\odot}$, and $30 M_{\odot}$ progenitor stars with 3
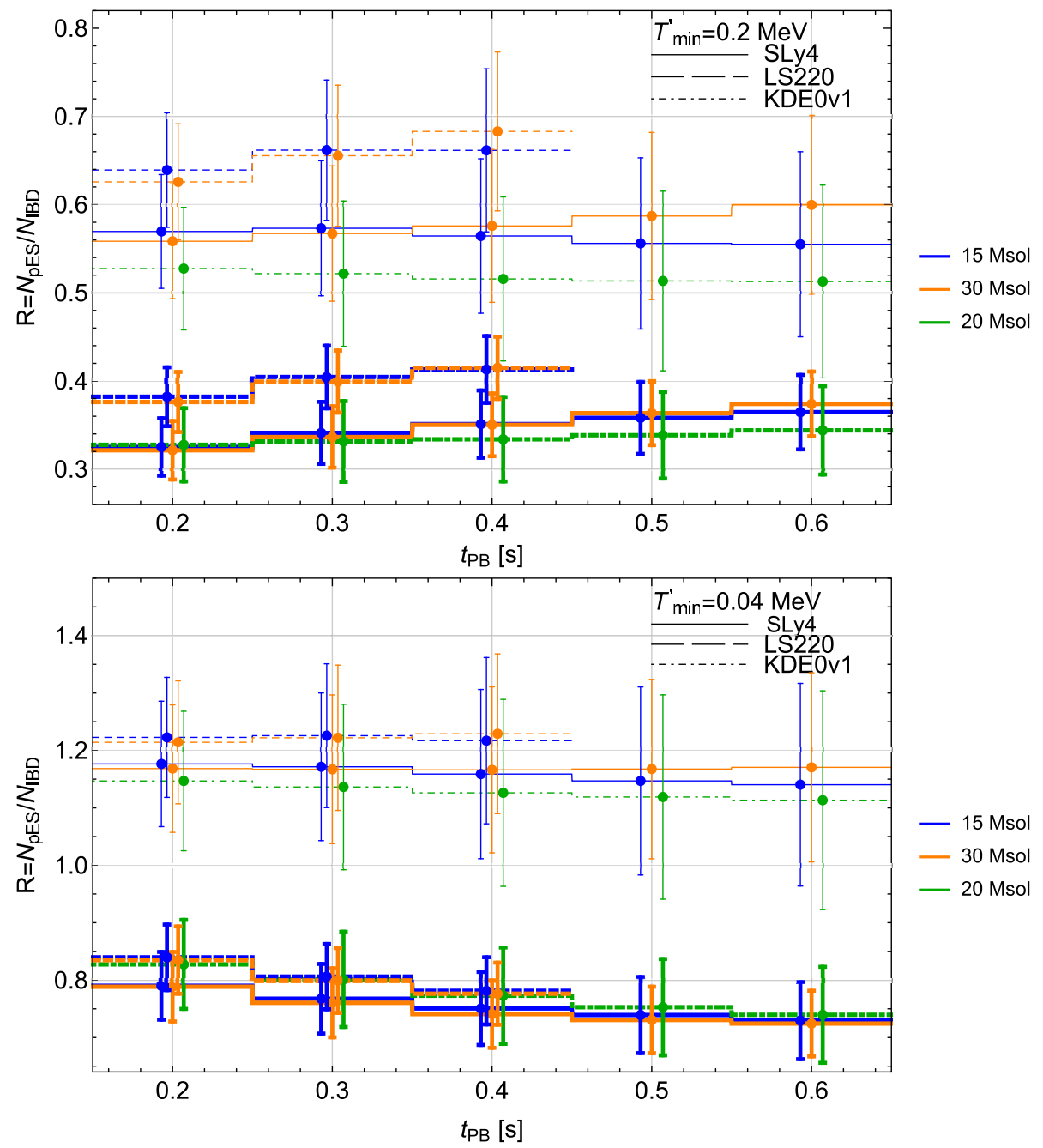

FIG. 8. Top: The observable $R$ vs postbounce time $t_{\mathrm{PB}}$, calculated for a galactic CCSN occurring $10 \mathrm{kpc}$ away and measured by JUNO with a reconstructed proton recoil energy threshold $T_{\min }^{\prime}=0.2 \mathrm{MeV}$. Thick lines (all occurring below $R=0.5$ ) correspond to a direct simulation of CITE. Thin lines (all occurring above $R=0.5$ ) correspond to the PNS cooling limit of the D $\nu \mathrm{M}$, explained in the text. Based on the $15 M_{\odot}, 20 M_{\odot}$, and $30 M_{\odot}$ progenitor star GR1D simulations, with 3 different assumed nuclear EoS. Error bars denote $1 \sigma$ statistical uncertainties. Calculated assuming adiabatic matter-induced flavor conversions with normal neutrino mass hierarchy. Bottom: same as the top panel, but with reconstructed proton recoil energy threshold $T_{\min }^{\prime}=0.04 \mathrm{MeV}$. 
different assumed nuclear EoS [72]: SLy4 [71], LS220 [73], and KDE0v1 [74]. Error bars denote $1 \sigma$ statistical uncertainties. We assume adiabatic matter-induced flavor conversions with normal neutrino mass hierarchy.

The bottom panel of Fig. 8 repeats the calculation of the top panel, but this time assuming $T_{\min }^{\prime}=0.04 \mathrm{MeV}$. The low threshold allows significantly better separation of CITE from bare PNS cooling, the separation occurring at $R \approx 0.9$, instead of $R \approx 0.5$ found in the upper panel. Importantly, low $T_{\min }^{\prime}$ causes the prediction of CITE to become less sensitive to progenitor mass and EoS, an important result that is decoupled from uncertainties related to our indirect estimate of the $\mathrm{D} \nu \mathrm{M}$.

In Fig. 9 we integrate the rates over the time interval $t_{\mathrm{PB}}=(0.2,0.65) \mathrm{sec}$, and explore the dependence on neutrino oscillation parameters by plotting the result vs the $\bar{\nu}_{e}$ survival probability $P_{\bar{e} \bar{e}}$. The top panel shows the results assuming $T_{\min }^{\prime}=0.2 \mathrm{MeV}$ and the bottom panel shows the results for $T_{\min }^{\prime}=0.04 \mathrm{MeV}$. Vertical error bars show $2 \sigma$ statistical uncertainties. Thin vertical lines around $P_{\bar{e} \bar{e}} \approx 0.68$ and $P_{\bar{e} \bar{e}} \approx 0.02$ show the allowed region of $P_{\bar{e} \bar{e}}$ assuming $\mathrm{NH}$ and $\mathrm{IH}$, respectively, with respect to the current $2 \sigma$ allowed range of the oscillation parameters.

We can now appreciate the sensitivity of $R$ to the details of neutrino flavor conversion: to determine the prediction in CITE, the neutrino mass hierarchy would be a crucial ingredient. Fortunately, there are good prospects for determining the hierarchy in the relatively near future (see, e.g., [112]). In fact, JUNO itself can deliver this information within the next decade [46]. Figure 9 shows that (i) given
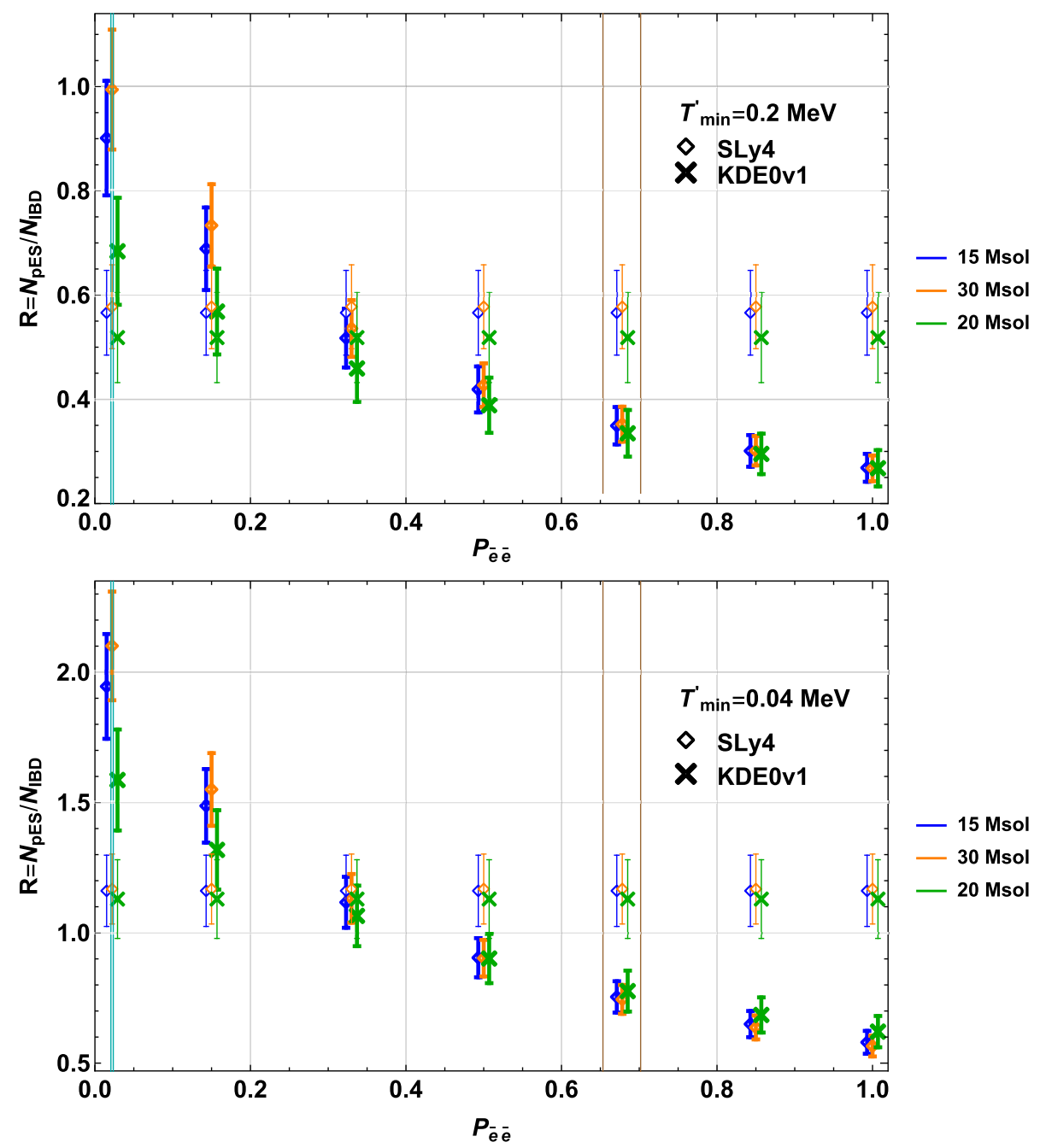

FIG. 9. Top: The observable $R$ vs the conversion probability $P_{\bar{e} \bar{e}}$, calculated for a galactic CCSN occurring 10 kpc away and measured by JUNO with a reconstructed proton recoil energy threshold $T_{\min }^{\prime}=0.2 \mathrm{MeV}$. The counts are obtained by integrating the expected detection rates in the time interval $(0.2,0.65) \mathrm{sec}$ postbounce. Based on the $15 M_{\odot}, 20 M_{\odot}$, and $30 M_{\odot}$ progenitor star GR1D simulations described in the text. Error bars denote $2 \sigma$ statistical uncertainties. Thin vertical lines around $P_{\bar{e} \bar{e}}=0.02$ and $P_{\bar{e} \bar{e}}=0.068$ highlight the allowed region of $P_{\bar{e} \bar{e}}$, obtained by varying the neutrino oscillation parameters within their current $2 \sigma$ range, for adiabatic matter-induced oscillations with IH and NH, respectively. Results for CITE (PNS cooling) are shown by thick (thin) markers. Bottom: same as in the top panel, but with reconstructed proton recoil energy threshold $T_{\min }^{\prime}=0.04 \mathrm{MeV}$. 
the binary information of $\mathrm{NH}$ vs $\mathrm{IH}$, further uncertainty due to the neutrino oscillation parameters - even with the current state of the art, not accounting for future improvement-would not limit the CCSN analysis; and (ii) the NH scenario would be particularly convenient.

Finally, we comment about the time integration in the computation of $R$, which we limited to the interval $t_{\mathrm{PB}}=(0.2,0.65) \mathrm{sec}$. The lower end of this interval was motivated by $\mathrm{D} \nu \mathrm{M}$ simulations, which tend to show explosions that start by approximately this time. $\mathrm{D} \nu \mathrm{M}$ explosion is needed to expel the accretion flow and terminate the accompanied accretion luminosity. Only after this happens, the D $\nu \mathrm{M}$ and the CITE scenarios begin to differ. The upper end was chosen in the interest of simulation run time. We note that CITE allows for a longer period of accretion luminosity, lasting up to a few seconds, with a possible termination due to $\mathrm{BH}$ formation and plausible subsequent continuation due to an accretion disk [35]. During this entire accretion period, CITE predicts $f_{\bar{e}} \gtrsim 2-3$ and a corresponding value of $R$ similar to the late time bins in Fig. 8. The actual statistical uncertainty on $R$, integrated on a longer period than that shown in Fig. 9, would therefore be better than in the plot.

\section{Sensitivity to modeling uncertainties.}

Assuming that the oscillation probability is known, our results from Sec. VA 1, based on GR1D simulations, suggest that measurements of $R$ can discriminate between CITE and the $\mathrm{D} \nu \mathrm{M}$, despite variances in progenitor and EoS details. It is important to analyze where this discrimination power comes from, and what are its limitations in terms of the sensitivity to the CCSN modeling. The neutrino transport implementation in GR1D (and other codes in the literature) is simplified, and does not include several complications that may modify the neutrino spectra in a realistic CCSN. In what follows, we study the sensitivity of our results to reasonable neutrino spectra that go beyond the range found in the numerical simulation. For concreteness, we focus on the $\mathrm{NH}$ adiabatic oscillations scenario, $P_{\bar{e} \bar{e}} \approx 0.68$.

For time-integrated spectra, the accretion-phase GR1D simulations are roughly consistent with the pinched Fermi-Dirac spectrum of Eq. (8). This is demonstrated in Fig. 10, using the KDE0v1 EoS [74] for a $20 M_{\odot}$ progenitor. The spectra are integrated over time in the range $t_{\mathrm{PB}}=(0.2,0.72) \mathrm{sec}$, and are normalized to the $\nu_{x}$ fluence at $E_{\nu}=1 \mathrm{MeV}$. Markers show the numerical result from the simulation. Solid lines show a fit to the pinched FermiDirac form of Eq. (8), with $a_{\nu_{x}} \approx-0.3,\left\langle E_{\nu_{x}}\right\rangle \approx 14.6 \mathrm{MeV}$; $a_{\bar{\nu}_{e}} \approx 0.7,\left\langle E_{\bar{\nu}_{e}}\right\rangle \approx 17.2 \mathrm{MeV} ; a_{\nu_{e}} \approx 0.35,\left\langle E_{\nu_{e}}\right\rangle \approx 14.3 \mathrm{MeV}$. The ratios of (time-averaged) luminosities are $L_{\bar{\nu}_{e}} / L_{\nu_{x}} \approx$ $2.74, L_{\bar{\nu}_{e}} / L_{\nu_{e}} \approx 1.03$.

To study the sensitivity of $R$ to modeling uncertainties, we therefore use Eq. (8) and vary the parameters $a_{\nu_{\alpha}},\left\langle E_{\nu_{\alpha}}\right\rangle$,

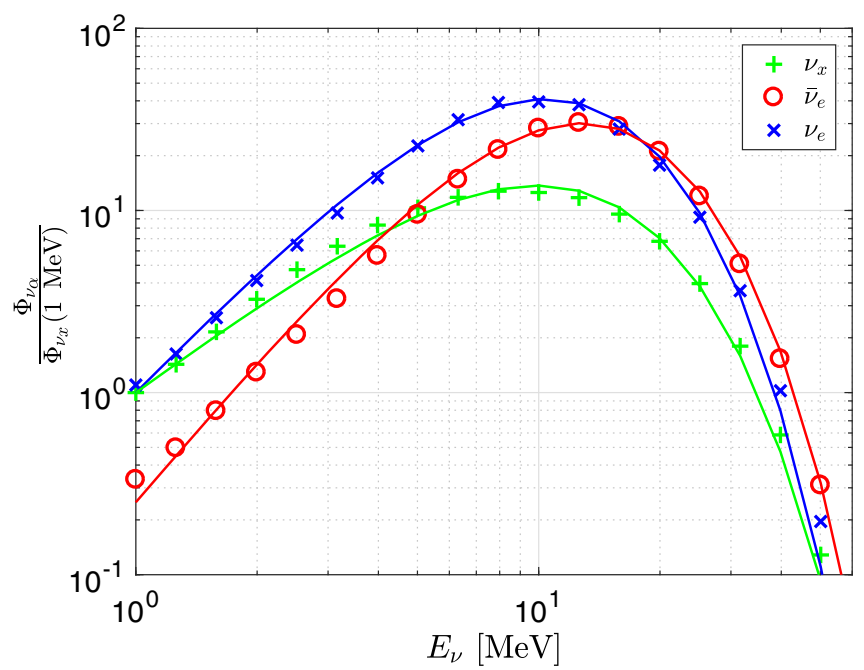

FIG. 10. Time-integrated neutrino spectra computed with GR1D, using the KDE0v1 [74] EoS for a $20 M_{\odot}$ progenitor. Markers show the numerical result from the simulation. Solid lines show a fit to the pinched Fermi-Dirac form of Eq. (8).

and $L_{\nu_{\alpha}}$ within a reasonable range. For simplicity, we fix the $\bar{\nu}_{e}$ and $\nu_{e}$ spectral parameters to be equal, setting $a_{\bar{\nu}_{e}}=a_{\nu_{e}}$, $\left\langle E_{\bar{\nu}_{e}}\right\rangle=\left\langle E_{\nu_{e}}\right\rangle$, and $L_{\bar{\nu}_{e}}=L_{\nu_{e}}$. We allow the $\nu_{x}$ parameters to vary independently from those of $\nu_{e}$, apart from the constraint $0.5<\left\langle E_{\nu_{x}}\right\rangle /\left\langle E_{\nu_{e}}\right\rangle<2$. Comparing to the numerical simulations, we note that the approximations $a_{\nu_{e}} \approx a_{\bar{\nu}_{e}}$ and $\left\langle E_{\nu_{e}}\right\rangle \approx\left\langle E_{\bar{\nu}_{e}}\right\rangle$ are, in fact, not very good: the GR1D $\nu_{e}$ spectra are softer than those of $\bar{\nu}_{e}$ (the approximation $L_{\bar{\nu}_{e}} \approx L_{\nu_{e}}$ appears, however, accurate). ${ }^{12}$ Nevertheless, relaxing the $\bar{\nu}_{e}-\nu_{e}$ spectral identification does not affect our results significantly.

In Fig. 11 we calculate $R$ as a function of the luminosity ratio $L_{\nu_{e}} / L_{\nu_{x}}$, using Eq. (8) as prescribed above and scanning over the range:

$a_{\nu_{x}}, a_{\nu_{e}}=(-0.5,2), \quad\left\langle E_{\nu_{x}}\right\rangle,\left\langle E_{\nu_{e}}\right\rangle=(15,30) \mathrm{MeV}$.

Each individual line in Fig. 11 corresponds to one set of the combination $\left\{a_{\nu_{x}}, a_{\nu_{e}},\left\langle E_{\nu_{x}}\right\rangle,\left\langle E_{\nu_{e}}\right\rangle\right\}$. Blue lines (generally corresponding to smaller numerical values for $R$ ) show the result for $\left\langle E_{\nu_{x}}\right\rangle=15 \mathrm{MeV}$. As $\left\langle E_{\nu_{x}}\right\rangle$ is increased, the numerical value of $R$ also increases; the magenta lines correspond to $\left\langle E_{\nu_{x}}\right\rangle=30 \mathrm{MeV}$. On the left, we show the result for $T_{\min }^{\prime}=0.2 \mathrm{MeV}$. On the right, we use $T_{\min }^{\prime}=0.04 \mathrm{MeV}$.

\footnotetext{
${ }^{12} \mathrm{~A}$ similar trend is seen, e.g., in the simulations of Ref. [75]. We suspect that the $\nu_{e}$ spectrum may be softer than the $\bar{\nu}_{e}$ spectrum, despite having almost equal luminosities, because of $\nu_{e} e^{-} \rightarrow \nu_{e} e^{-}$elastic scattering distorting the shape of the $\nu_{e}$ spectrum on its way out of the star, without appreciably affecting the total energy carried by it. However, we did not investigate this issue further.
} 

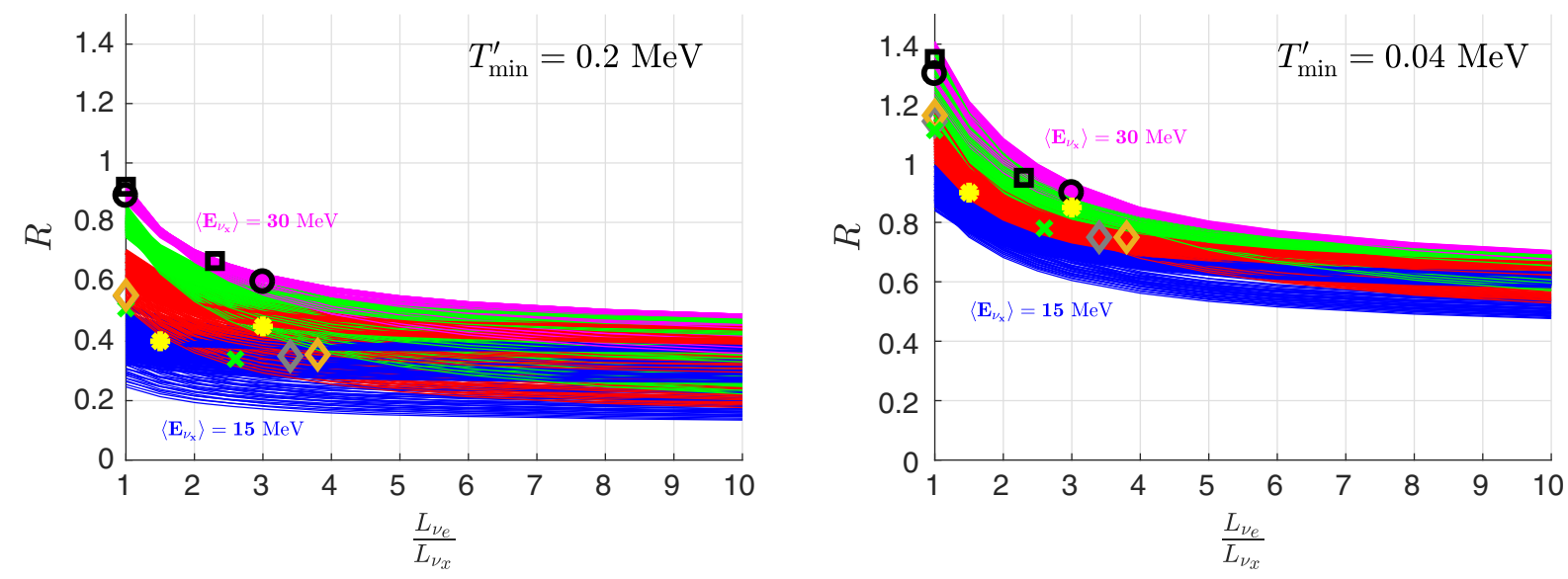

FIG. 11. $R$ vs the luminosity ratio $L_{\nu_{e}} / L_{\nu_{x}}$, calculated for the pinched Fermi-Dirac spectral parametrization of Eq. (8) with varying model parameters (see text for details). Left: lower (quenched) proton recoil energy threshold $T_{\min }^{\prime}=0.2 \mathrm{MeV}$. Right: $T_{\min }^{\prime}=0.04 \mathrm{MeV}$. The results of three GR1D calculations are shown, denoted by the same markers as in Fig. 9 with the some marker colors besides from for the $15 \mathrm{M}_{\odot}$ case, where the blue is replaced by grey for clarity in the plot. We also show estimated results from external simulations, discussed in Appendix A. Two results from Ref. [75] are shown by black circle (square) markers, denoting the HC (LC) progenitors there. Results from Ref. [7] are estimated by yellow star.

The results of three GR1D calculations are superimposed in Fig. 11, denoted by the same markers as in Fig. 9 with the same marker colors besides from for the $15 M_{\odot}$ case, where the blue is replaced by grey for clarity in the plot. We also show estimated results from external simulations [7,75], discussed in Appendix A. We do not have detailed neutrino spectra from these simulations. We therefore estimate $R$ for these codes by using the mean neutrino energies and luminosity ratios reported in $[7,75]$. Two results from Ref. [75] are shown by black circle (square) markers, denoting the HC (LC) progenitors there. ${ }^{13}$ Results from Ref. [7], approximately applicable to the 11, 17, and $19 M_{\odot}$ progenitors considered in Appendix A, are estimated collectively by yellow star.

We make a few comments regarding Fig. 11:

(i) At a given value of $L_{\nu_{e}} / L_{\nu_{x}}$, Fig. 11 shows a significant spread in $R$. The dominant variable that correlates with this spread is the neutrino mean energy $\left\langle E_{\nu}\right\rangle$, and the cause for the spread is the $\mathrm{pES}$ detection efficiency $\epsilon_{\mathrm{pES}}$ [see Eq. (9)]: the right panel of Fig. 6 shows that for $\left\langle E_{\nu}\right\rangle$ varying in the range 15-30 MeV, $\epsilon_{\mathrm{pES}}$ changes by a factor of $\sim 2$ for $T_{\min }^{\prime}=0.2 \mathrm{MeV}$, or a factor of $\sim 1.5$ for $T_{\min }^{\prime}=$ $0.04 \mathrm{MeV}$. The same effect is seen in the semianalytical calculation (solid lines) and the numerical simulations (markers). For example, the $\nu_{x}$ spectra from Ref. [75] are generically characterized by higher mean energy than predicted by GR1D, which brings these simulations to predict high values of $R$.

It is important to note, that the mean energy of the $\bar{\nu}_{e}$ component of the flux at Earth would be well

\footnotetext{
${ }^{13}$ The PUSH ("D $\nu \mathrm{M}$ ") simulations in [75] actually get $L_{\bar{\nu}_{e}} / L_{\nu_{x}}$ very near, but slightly smaller than unity.
}

determined by the IBD event spectra. This means that some of the $\left\langle E_{\nu}\right\rangle$-related spread in the $R$ vs $L_{\nu_{e}} / L_{\nu_{x}}$ relation can be mitigated by a spectral analysis, going beyond $R$ alone. We return to this point extensively below, in this and the next sections.

(ii) As noted earlier, the 2D "exploding" simulations of Ref. [7] demonstrate a deviation from the simple PNS cooling limit of the $\mathrm{D} \nu \mathrm{M}$ : the luminosity ratio found in these $2 \mathrm{D}$ simulations is $L_{\nu_{e}} / L_{\nu_{x}} \sim 1.5$, rather than $L_{\nu_{e}} / L_{\nu_{x}} \sim 1$. The reason for this behavior, as noted in [7], is that accretion onto the PNS is observed to continue, albeit at a reduced level, even after the $\mathrm{D} \nu \mathrm{M}$-driven explosion is ongoing.

We conclude that in order to mitigate modeling uncertainties, the consideration of $R$ needs to be combined with a spectral analysis of the IBD data, supplemented by the less precise information from the quenched $\mathrm{pES}$ recoil energy spectrum.

The parameter reconstruction exercise is illustrated in Fig. 12. Here, we show detected energy spectra of IBD and pES for the $20 M_{\odot}$ GR1D simulation (the same simulation that was used in Fig. 10). On the left panel we show the CITE scenario. On the right we show the PNS cooling scenario. The IBD peak is seen at $E_{d} \sim 20 \mathrm{MeV}$, while the pES quenched energy spectrum peaks at $E_{d} \sim 0.1 \mathrm{MeV}$. The two pES lower threshold values discussed in this work, $T_{\min }^{\prime}=0.04$ and $0.2 \mathrm{MeV}$, are marked by solid vertical lines. The pES upper threshold $T_{\max }^{\prime}=2 \mathrm{MeV}$ is marked by a dashed vertical line. The contributions due to individual neutrino flavors are shown in green, red, and blue for $\nu_{x}, \bar{\nu}_{e}$, and $\nu_{e}$, respectively. These colors correspond to neutrino flavor at the source, before oscillations are taken into account (with $P_{\bar{e} \bar{e}}=0.68$ ). 

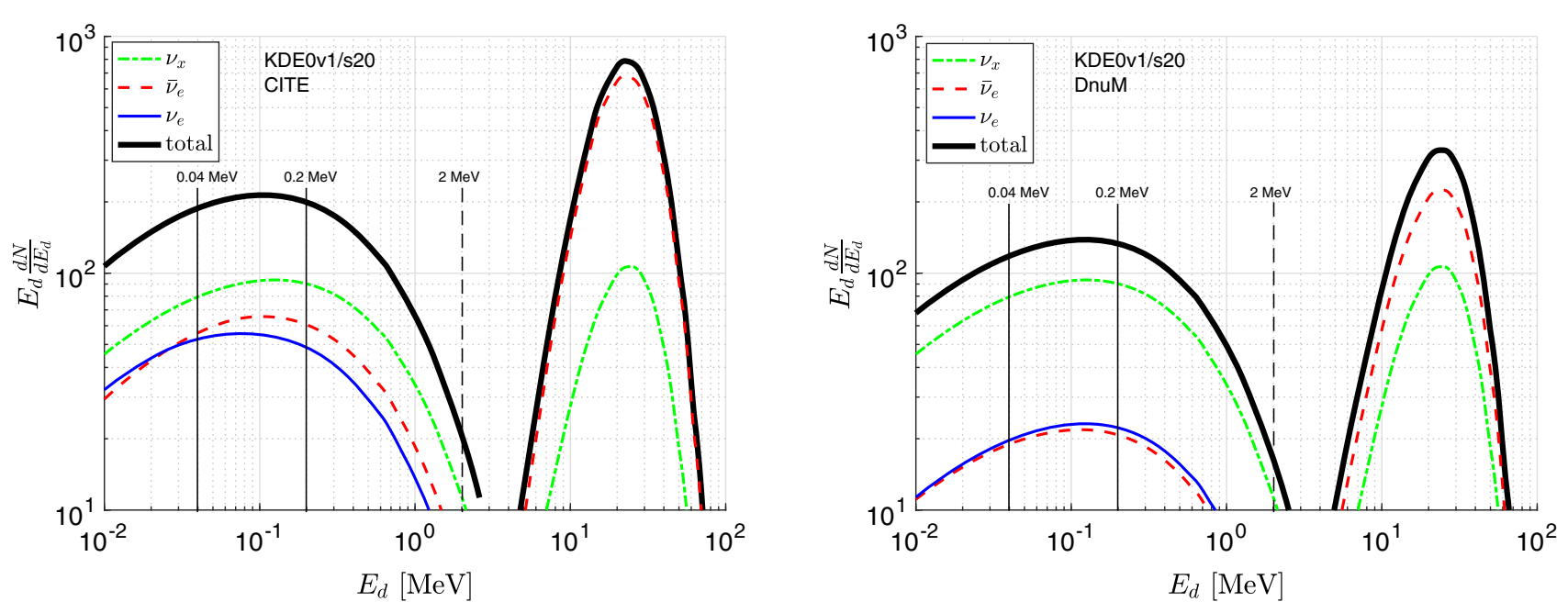

FIG. 12. Time-integrated deposited energy spectra, for the simulation shown in Fig. 10. IBD peaks at $E_{d} \sim 20 \mathrm{MeV}$, while pES peaks at $E_{d} \sim 0.1 \mathrm{MeV}$. The pES spectra are quenched. Left: CITE scenario. Right: PNS cooling limit of the D $\nu \mathrm{M}$ (estimated by setting the flux of $\nu_{e}$ and $\bar{\nu}_{e}$ equal to the simulated flux of $\nu_{x}$ ). The $\mathrm{pES}$ lower thresholds are marked by solid vertical lines. The pES upper threshold is marked by a dashed vertical line.

Considering Fig. 12, the problem of diagnosing the emission process boils down to telling apart between the left and the right panels. The name of the game is to find the correct balance between the $\nu_{x}$ (green) and $\bar{\nu}_{e}$ (red) contributions. Because of modeling uncertainties, this should better be done without imposing strong theoretical bias on the individual parametrization of each of the spectra. Note that accurate reconstruction of the $\nu_{e}$ contribution, which only affects pES, is not crucial; setting the $\nu_{e}$ spectrum to match that of $\bar{\nu}_{e}$ is a reasonable approximation that does not affect the results. Further, our analysis in this and the previous sections suggests that if the mean energies of $\nu_{x}$ and $\bar{\nu}_{e}$ can be deduced from the data with precision of a few tens of percent or better, than the pES/IBD event count ratio (with appropriate, not too high, threshold $T_{\min }^{\prime}$ ) provides the remaining information needed to determine the $L_{\bar{\nu}_{e}} / L_{\nu_{x}}$ luminosity ratio, and thus identify accretion-dominated emission. We explore this reconstruction problem in the next section.

\section{B. Likelihood analysis}

In this section we go beyond simple counting, and attempt to reconstruct directly $L_{\bar{\nu}_{e}} / L_{\nu_{x}}$ from the experimental information. We base our analysis on GR1D as a concrete example. Given a numerical simulation, we construct multiple mock realizations of deposited energy spectra collected over a time interval $\Delta t$ at JUNO, including the effects of detector energy resolution and analysis cuts. For each of the mock detections, using the unbinned Poisson likelihood method of $[33,34,113]$, we fit a pinched Fermi-Dirac model as in Eq. (8). We keep the fit parameters constant during the time interval $\Delta t$; time-dependent information can be obtained by analyzing different time segments along the burst.

For simplicity, in fitting the mock data, we set equal the $\bar{\nu}_{e}$ and the $\nu_{e}$ spectra: $L_{\nu_{e}}=L_{\bar{\nu}_{e}}, a_{\nu_{e}}=a_{\bar{\nu}_{e}}, T_{\nu_{e}}=T_{\bar{\nu}_{e}}$. We stress that this simplification, just like the simplified spectral form given in Eq. (8), is taken only in the fitting procedure and not in the calculation of the mock detection data being fitted. The mock data uses the full flavor- and energy-dependent information from the simulations. We thus have in total 6 free parameters, $\left\{L_{\nu_{x}}, a_{\nu_{x}}, T_{\nu_{x}}\right\}$ and $\left\{L_{\bar{\nu}_{e}}, a_{\bar{\nu}_{e}}, T_{\bar{\nu}_{e}}\right\}$.

We present results in terms of the distribution of reconstructed best-fit parameters. Specifically, we focus on the ratio of best-fit $\bar{\nu}_{e}$ and $\nu_{x}$ luminosity parameters, $\left(L_{\bar{\nu}_{e}} / L_{\nu_{x}}\right)_{\mathrm{BF}}$. This distribution captures the key information we are after, considering $\left(L_{\bar{\nu}_{e}} / L_{\nu_{x}}\right)_{\mathrm{BF}}$ as one possible teststatistic with direct physical interpretation.

Figure 13 shows the probability distribution (PDF) of $\left(L_{\bar{\nu}_{e}} / L_{\nu_{x}}\right)_{\mathrm{BF}}$ obtained for 150 mock data realizations with CITE (blue) and with the PNS cooling limit of the D $\nu \mathrm{M}$ (orange). The neutrino source is a GR1D simulation for a 30 and $20 M_{\odot}$ star (top and bottom panels, respectively). The analyzed time segment was [0.2,0.7] sec postbounce. In the left (right) panel, the proton deposited recoil energy threshold was $T_{\min }^{\prime}=0.2 \mathrm{MeV}(0.04 \mathrm{MeV})$. Thick lines show the result when setting the distance to the CCSN to $D_{\mathrm{SN}}=10 \mathrm{kpc}$. Thin lines show the result when the distance is $D_{\mathrm{SN}}=3 \mathrm{kpc}$; equivalently, these results correspond to a detector with 9 times the effective volume of JUNO. We assume $P_{\bar{e} \bar{e}}=0.68$.

The likelihood modeling of Figs. 13 generalizes the simple analysis of the counting observable $R$. Without imposing strong modeling bias, and despite degeneracies 

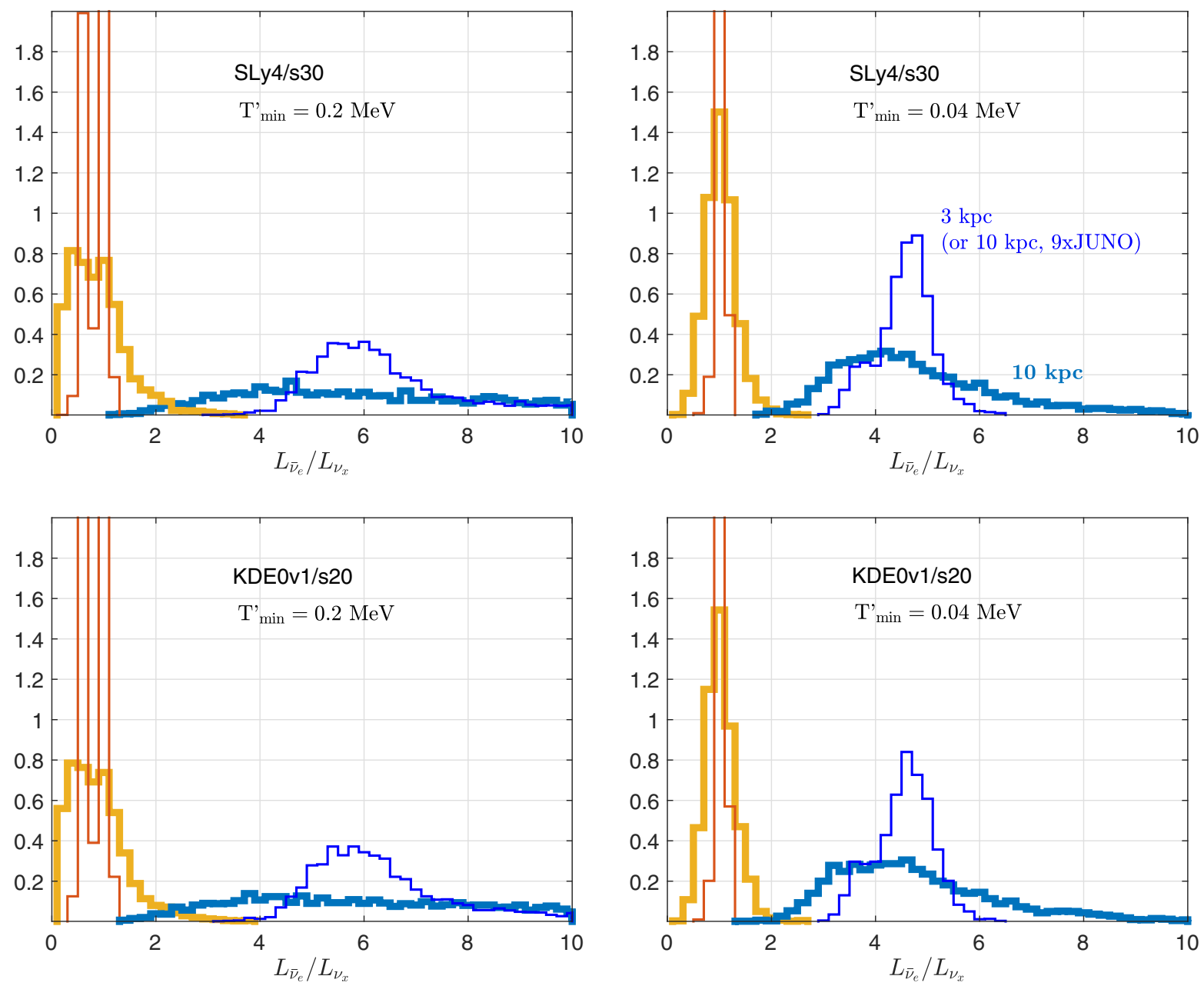

FIG. 13. Distribution of reconstructed best-fit luminosity ratios for 150 mock data realizations, computed from GR1D simulations of CITE (blue) and the PNS cooling limit of the D $\nu \mathrm{M}$ (orange). Top, Bottom: 30 and $20 M_{\odot}$ progenitors, respectively. The analyzed time interval is [0.2-0.7] sec postbounce. Left: Proton recoil deposited energy threshold $T_{\min }^{\prime}=0.2 \mathrm{MeV}$. Right: $T_{\text {min }}^{\prime}=0.04 \mathrm{MeV}$.

in the neutrino spectral parameters, a global analysis of the deposited energy spectra at JUNO within the first $\sim 1 \mathrm{sec}$ of the CCSN burst would allow us to constrain the luminosity ratio $L_{\bar{\nu}_{e}} / L_{\nu_{x}}$. Accretion-dominated emission with $L_{\bar{\nu}_{e}} / L_{\nu_{x}} \gtrsim 3$ can be discriminated from $L_{\bar{\nu}_{e}} / L_{\nu_{x}}=1$ with high confidence. The analysis in Sec. VA 2 makes clear how this discrimination works: (i) the IBD spectrum effectively constrains the mean neutrino energies; (ii) the pES/IBD event ratio then allows to reconstruct $L_{\bar{\nu}_{e}} / L_{\nu_{x}}$, with relative insensitivity to details of the spectral pinch parameter $\left[a_{\nu}\right.$ in Eq. (8)]. Degeneracy towards high values of $L_{\bar{\nu}_{e}} / L_{\nu_{x}} \gg 1$ is seen, and is understood from, e.g., Fig. 11: once $L_{\bar{\nu}_{e}} / L_{\nu_{x}} \gtrsim 3$ is achieved, the pES/IBD ratio approaches its asymptotic value obtained at $L_{\bar{\nu}_{e}} / L_{\nu_{x}} \rightarrow \infty$. This asymptotic value, $R \rightarrow 2 \times \frac{1.7}{6.1} \frac{\epsilon_{\mathrm{PES}}}{P_{\bar{e} \bar{e}}}$ for $L_{\bar{\nu}_{e}} / L_{\nu_{x}} \rightarrow \infty$, can be understood from the approximate cross section formula in Eqs. (B6)-(B7), combined with the pES efficiency $\epsilon_{\mathrm{pES}}$ shown in the right panel of Fig. 6.

\section{SUMMARY}

Most of the theoretical efforts in solving the explosion mechanism of core-collapse supernovae (CCSNe) revolve around the hypothesis, that a small fraction (of order percent) of the large gravitational binding energy of the core (few $10^{53} \mathrm{erg}$ ) couples somehow to the stellar envelope and powers the explosion (with kinetic energy $E_{K} \sim 10^{51}-10^{52} \mathrm{erg}$ ). The delayed neutrino mechanism $(\mathrm{D} \nu \mathrm{M})$ is the most well studied example of this kind [1-4]. A competing hypothesis is that the supernova is powered by a collapse-induced thermonuclear explosion of the envelope [21-25] (CITE). In this case, the explosion energy does not come from a low-efficiency coupling of the envelope to the core. Instead, the explosion energies of $\mathrm{CCSNe}$ are reproduced by the $\sim \mathrm{MeV}$ nuclear binding energy per nucleon, released in burning a few $M_{\odot}$ of the progenitor star [26], with $\mathcal{O}(1)$ efficiency.

The explosion timescale in the $\mathrm{D} \nu \mathrm{M}$ is of the order of the dynamical timescale of the $\sim 1-2 M_{\odot}$ inner core, less than 
$1 \mathrm{sec}$. In contrast, in CITE the explosion timescale is of the order of the dynamical timescale of the He-O layer, about $10 \mathrm{sec}$. As a result, the D $\nu \mathrm{M}$ predicts a short period of accretion-dominated neutrino luminosity, transiting at $t<1 \mathrm{sec}$ into dominant protoneutron star (PNS) cooling (possibly with some residual accretion), while CITE predicts a prolonged accretion phase that can last for a few sec.

In both models, the total neutrino luminosity could vary significantly with time on hundreds of ms timescale. Such variations follow from the accretion of composition transition layers through the PNS accretion shock. In the $\mathrm{D} \nu \mathrm{M}$, a luminosity drop occurs upon explosion when the accretion component is blown away. In CITE, a feature could follow from $\mathrm{BH}$ and/or accretion disk formation. Both models also exhibit nontrivial time evolution of the mean neutrino energy. In the $\mathrm{D} \nu \mathrm{M}$, neutrino energies rise during the first $\sim 1-2$ sec but then decrease smoothly as the PNS cools. In CITE, neutrino energies are expected to rise toward $\mathrm{BH}$ formation, but the formation of a dominant accretion component from an accretion disc could lead the observed mean energy to decrease. In case of a galactic CCSN, these different features would be manifest with high statistics and could help to diagnose the explosion mechanism. In particular, a sharp drop in total luminosity accompanied by a few sec of quiescence, as a result of $\mathrm{BH}$ formation, would allow clean identification of a CITElike scenario. Such abrupt luminosity gap is consistent with data for SN1987A [35], but statistics for that event were too sparse to allow robust conclusions.

It is possible, however, that the next galactic CCSN would not provide us with a clear smoking gun like prompt BH formation followed by a luminosity gap; and the various luminosity and energy features discussed above could be nontrivial to interpret. In this work, we therefore focused on another characteristic-the relative neutrino flavor composition at the source-as a diagnostic of the explosion. Accretion-dominated neutrino emission at the source is characterized by enhanced $\nu_{e}, \bar{\nu}_{e}$ compared to $\nu_{x}$ (where $x$ stands collectively for $\mu$ and $\tau$ neutrinos and antineutrinos). Focusing on the scintillation detector JUNO, currently under construction, we showed that the ratio $R=\mathrm{pES} / \mathrm{IBD}$ between inverse-beta decay (IBD) and proton-neutrino elastic scattering (pES) event rates-the two dominant detection channels, assuming realistic detection energy thresholds-is a robust probe of such an accretion-dominated source. Using 1D numerical simulations, we studied the impact of progenitor star profile and of nuclear equation of state (EoS) on the source prediction. Given that the mean neutrino energy can be reconstructed with $\mathcal{O}(10 \%)$ accuracy, from the spectrum of detected IBD events, we find that the accretion-dominated scenario makes a distinctive prediction for $R$. Theoretical model uncertainties shrink if we allow optimistic lower quenched proton energy threshold, $T_{\min }^{\prime}<0.1 \mathrm{MeV}$, as compared to the nominal $T_{\min }^{\prime}=0.2 \mathrm{MeV}$ currently suggested for JUNO. The main caveats in the analysis are the unknown impact of neutrino self-induced flavor oscillations at the source, and the current uncertainty on the $\mathrm{pES}$ cross section.

\section{ACKNOWLEDGMENTS}

We are grateful to Evan O'Connor and Christian Ott for making their numerical simulation code GR1D publicly available, and to Evan O'Connor for help in technical issues related to running the code. We thank Avital Dery, Yossi Nir, and Eli Waxman for useful discussions, John Beacom for comments on the manuscript, and Doron Kushnir for many insightful discussions and for providing MESA profiles and CITE numerical simulation data. The work of N. B. and K. B. was supported by Grant No. 1937/12 from the I-CORE program of the Planning and Budgeting Committee and the Israel Science Foundation and by Grant No. 1507/16 from the Israel Science Foundation. K. B. is incumbent of the Dewey David Stone and Harry Levine career development chair. G.D. A. is supported by the Simons Foundation Origins of the Universe program (Modern Inflationary Cosmology collaboration).

\section{APPENDIX A: COMPARING ACCRETION- DOMINATED AND PNS COOLING-DOMINATED NEUTRINO EMISSION IN DIFFERENT SIMULATIONS}

In the body of this work, we used GR1D to simulate the accretion phase of a CCSN neutrino burst. If CITE causes the explosion, then this "accretion phase" luminosity with its characteristic neutrino flavor pattern is expected to describe the entire neutrino burst, with a possible temporary pause on $\mathrm{BH}$ formation [35].

On the other hand, because the 1D simulation does not explode, we cannot directly simulate the neutrino flavor content of the $\mathrm{D} \nu \mathrm{M}$. This caveat could only be truly resolved with numerically converged, demonstrated $\mathrm{D} \nu \mathrm{M}$ explosions. Until such tools become available, below we show two $\mathrm{D} \nu \mathrm{M}$ examples from the literature where explosions are either started artificially by hand, or where explosions are reported albeit with no demonstration of numerical convergence.

\section{1. "PUSH" simulations of Ref. [75]}

Reference [75] reported the results of state of the art simulations in which artificial " $\mathrm{D} \nu \mathrm{M}$ explosions" were triggered by hand. Conveniently for our purpose, Ref. [75] also reported, for the same initial conditions, the results of simulation runs in which the explosion was not triggered, allowing to demonstrate the difference in flavor content between the two cases. The results are shown in Fig. 14, where the left and right panels depict two different progenitor star profiles, denoted high compactness (HC) 

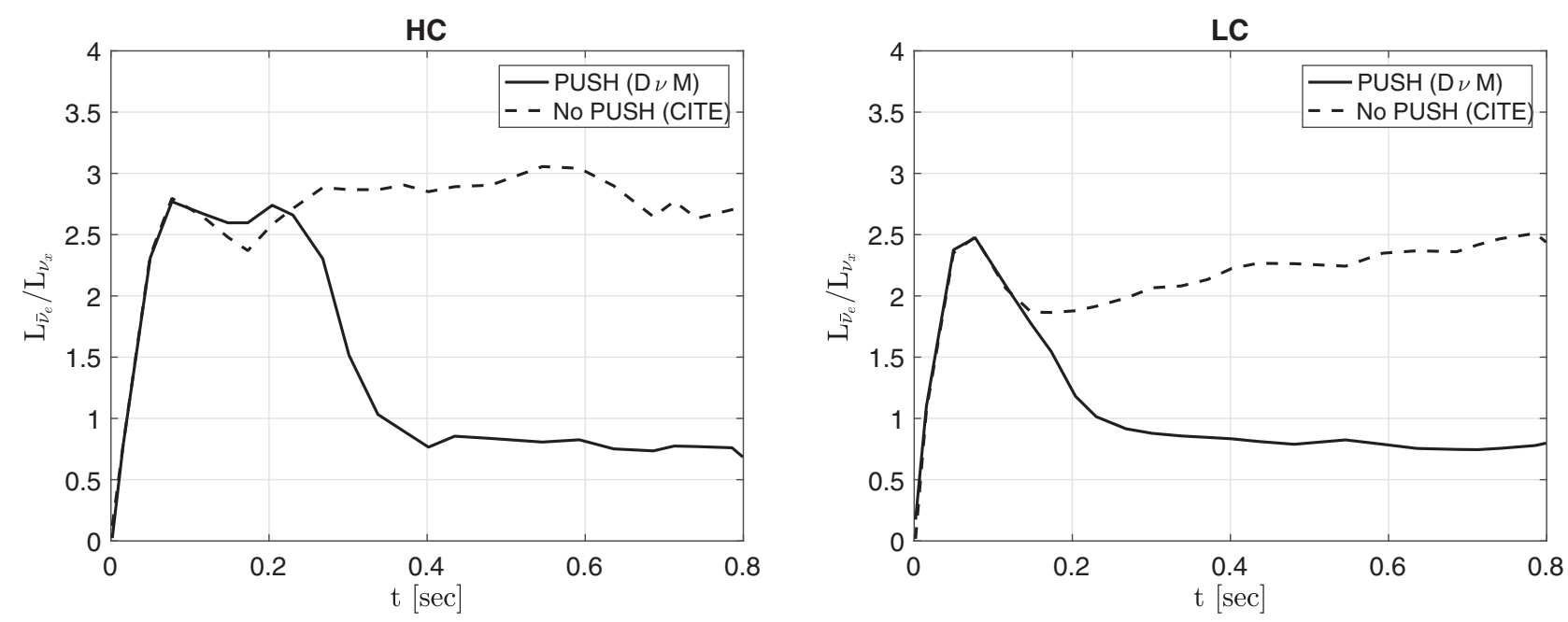

FIG. 14. Temporal dependence of the neutrino luminosity, taken from the simulations of Ref. [75]. Left: The HC progenitor of [75]. Right: The LC progenitor of [75]. In both panels, solid curves (denoted PUSH) show the case where an artificial explosion is triggered by hand at $t \sim 0.2 \mathrm{sec}$, while dashed curves (denoted No PUSH) show the result where the simulation is allowed to run without modification, so that no explosion occurs within the simulated time. The solid curve demonstrates the prediction of the $\mathrm{D} \nu \mathrm{M}$, while the dashed curve demonstrates the prediction of CITE.

and low compactness (LC) in [75]. These progenitor profiles correspond to zero-age main sequence (ZAMS) mass of 20 and $19.2 M_{\odot}$, respectively.

\section{2. "1D nonexplosion" vs "2D exploding" simulations of Ref. [7]}

Reference [7] studied the neutrino signal of a galactic CCSN, highlighting - as we did here - the signature of the accretion phase. In [7], 1D and 2D simulations of the same progenitor star were performed. The $2 \mathrm{D}$ simulations where reported as exploding, while the $1 \mathrm{D}$ simulations did not explode. These two sets of simulations give a useful demonstration of the flavor information we study in this

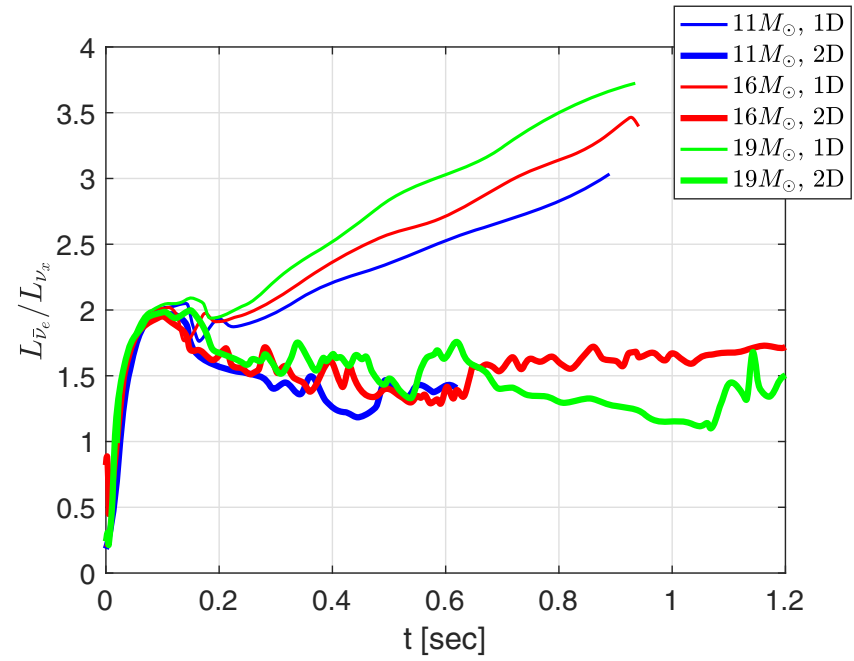

FIG. 15. Temporal dependence of $f_{\bar{e}}=L_{\bar{\nu}_{e}} / L_{\nu_{x}}$, calculated from the published plots in [7]. work. In Fig. 15 we show the $L_{\bar{\nu}_{e}} / L_{\nu_{x}}$ ratio calculated from the published plots in [7] for three progenitor masses.

\section{APPENDIX B: NEUTRINO SCATTERING CROSS SECTIONS}

The differential cross section for neutrino-proton elastic scattering (pES) with proton recoil energy $T$ and initial neutrino energy $E_{\nu}$, is given by [114]

$$
\begin{aligned}
\frac{d \sigma_{\nu(\bar{\nu}) p}}{d T}= & \frac{G_{F}^{2} m_{p}}{2 \pi}\left(\left(c_{V p} \pm c_{A p}\right)^{2}+\left(c_{V p} \mp c_{A p}\right)^{2}\left(1-\frac{T}{E_{\nu}}\right)^{2}\right. \\
& \left.+\left(c_{A p}^{2}-c_{V p}^{2}\right) \frac{m_{p} T}{E_{\nu}^{2}} \pm 4 c_{M p} c_{A p}\left(1 \pm \xi_{M p}\right) \frac{T}{E_{\nu}}\right), \\
c_{V p}= & \frac{1-4 s_{W}^{2}}{2}, \quad c_{A p}=\frac{1.27-\Delta s}{2}, \quad c_{M p}=\frac{\mu_{p}}{2} \approx 1.4, \\
\xi_{M p}= & \frac{T}{E_{\nu}} \frac{c_{V p}+\frac{1}{4} c_{M p} \mp c_{A p}}{c_{A p}}+\frac{c_{M p}}{2 c_{A p}}\left(1-\frac{T}{E_{\nu}}\right) \frac{E_{\nu}}{m_{p}} .
\end{aligned}
$$

Here, in \pm , the upper sign refers to $p+\nu \rightarrow p+\nu$ and the lower sign refers to $p+\bar{\nu} \rightarrow p+\bar{\nu}$. For the Weinberg angle we use $s_{W}^{2}=0.23$ [115]. For the $\Delta s$ correction in the expression for $c_{A p}$, due to the strange quark contribution to the proton spin, a recent result from [81] is $\Delta s=-0.196 \pm$ 0.127 . The uncertainty on $\Delta s$ leads to an uncertainty of $\approx \pm 9 \%$ on $c_{A p}$ which, in turn, implies a systematic cross section uncertainty of $\approx \pm 20 \%$. This would be a large systematic effect, directly entering the $\mathrm{pES} / \mathrm{IBD}$ ratio. There is a proposal to reduce this uncertainty by an order of magnitude [51], and as explained in the main text we 
assume that the uncertainty on $\sigma_{\mathrm{pES}}$ can indeed be reduced to the few percent level. Since the uncertainty is still very large, we use for concreteness $\Delta s=0$ in all calculations.

The maximal proton recoil energy is

$$
T_{\max }=\frac{2 E_{\nu}^{2}}{m_{p}+2 E_{\nu}}
$$

implying $T / E_{\nu}<2 E_{\nu} / m_{p} \ll 1$ for CCSN neutrinos. In all of the core-collapse simulations we study, neutrinos with $E_{\nu}>100 \mathrm{MeV}$ contribute no more than a few percent of the detected events. For $E_{\nu}=100 \mathrm{MeV}, T / E_{\nu}<0.2$, implying sub-percent contribution to the detected events coming from terms in Eq. (B1) that are linear in $T / E_{\nu}$ (not including the third term in Eq. (B1), where the factor $T / E_{\nu}$ is compensated by a factor $m_{p} / E_{\nu}$ ). The weak magnetism contribution, proportional to $c_{M p} c_{A p}$, is suppressed both by an uncompensated factor of $T / E_{\nu}$ and in addition by the fact that it reverses sign (up to the numerically insignificant $\xi_{M p}$ correction) when changing between $\nu$ and $\bar{\nu}$. Corecollapse simulations and analytic estimates predict nearly equal spectra for $\nu_{\mu, \tau}$ and $\bar{\nu}_{\mu, \tau}$ (which, therefore, are usually collectively denoted $\nu_{x}$ ), such that the weak magnetism contributions cancel out for the $\nu_{x}$ part of the flux. The $\nu_{e}$ and $\bar{\nu}_{e}$ spectra are not equal, but are still observed to follow each other to about $20 \%$ level in simulations (this discussion pertains to the postbounce phase of the collapse).

The cross section for inverse beta decay (IBD), $p+\bar{\nu}_{e} \rightarrow n+e^{+}$, is given to $\sim 1 \%$ accuracy by [116]

$$
\begin{gathered}
\sigma_{\mathrm{IBD}}=\kappa_{\mathrm{IBD}} p_{e} E_{e}, \\
\kappa_{\mathrm{IBD}}=10^{-43} \exp \left(-0.07056 x+0.02018 x^{2}\right. \\
\left.-0.001953 x^{4}\right) \mathrm{cm}^{2},
\end{gathered}
$$

where $p_{e}=\sqrt{E_{e}^{2}-m_{e}^{2}}$ and $x=\log \left(E_{\nu} / \mathrm{MeV}\right)$.

The cross section for neutrino-electron elastic scattering, $e^{-}+\nu \rightarrow e^{-}+\nu$, depends on the neutrino flavor. For $1<E_{\nu}<100 \mathrm{MeV}$, to $\sim 10 \%$ accuracy, it is given by (see, e.g., [117])

$$
\begin{gathered}
\sigma_{\nu_{e} e}=1.86 \times 10^{-43}\left(\frac{E_{\nu}}{20 \mathrm{MeV}}\right) \mathrm{cm}^{2}, \\
\sigma_{\bar{\nu}_{e} e}=0.78 \times 10^{-43}\left(\frac{E_{\nu}}{20 \mathrm{MeV}}\right) \mathrm{cm}^{2}, \\
\sigma_{\nu_{\mu, \tau} e}=0.32 \times 10^{-43}\left(\frac{E_{\nu}}{20 \mathrm{MeV}}\right) \mathrm{cm}^{2}, \\
\sigma_{\bar{\nu}_{\mu, \tau} e}=0.26 \times 10^{-43}\left(\frac{E_{\nu}}{20 \mathrm{MeV}}\right) \mathrm{cm}^{2} .
\end{gathered}
$$

For $E_{\nu}$ between 10 to $50 \mathrm{MeV}$, the total $\mathrm{pES}$ and IBD cross sections are well approximated by

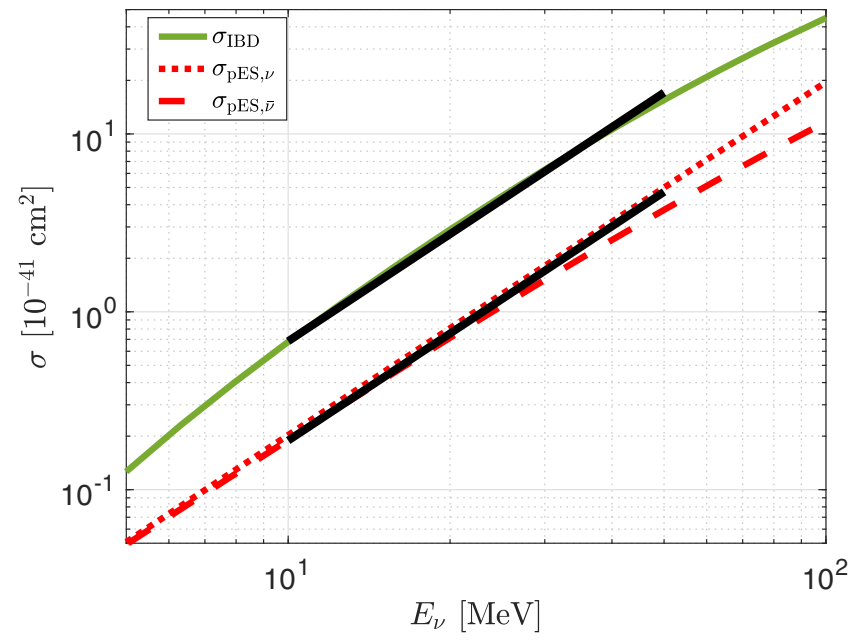

FIG. 16. Total pES and IBD cross sections. The approximate formulae of Eqs. (B6)-(B7) are shown by black lines. This plot is done using $\Delta s=0$.

$$
\begin{gathered}
\sigma_{\mathrm{pES}} \approx 1.7 \times 10^{-41}\left(\frac{E_{\nu}}{30 \mathrm{MeV}}\right)^{2}(1-1.5 \Delta s), \\
\sigma_{\mathrm{IBD}} \approx 6.1 \times 10^{-41}\left(\frac{E_{\nu}}{30 \mathrm{MeV}}\right)^{2} .
\end{gathered}
$$

The IBD and pES total cross sections are shown in Fig. 16. Here, for concreteness, we use $\Delta s=0$.

\section{APPENDIX C: ON THE ADIABATICITY CONDITION FOR NEUTRINO OSCILLATIONS IN AN ACCRETION-DOMINATED CCSN NEUTRINO BURST}

In the absence of self-induced oscillations, the propagation of neutrinos in matter reduces to a Schrödinger equation describing the flavor transformation of the wave function of a neutrino with energy $E$ along the radial direction (for a review, see, e.g., [118]),

$$
\begin{aligned}
i \frac{d}{d r}\left(\begin{array}{c}
\nu_{e} \\
\nu_{\mu} \\
\nu_{\tau}
\end{array}\right)= & {\left[\frac{1}{2 E} U\left(\begin{array}{ccc}
-\delta m^{2} / 2 & 0 & 0 \\
0 & \delta m^{2} / 2 & 0 \\
0 & 0 & \pm \Delta m^{2}
\end{array}\right) U^{\dagger}\right.} \\
& \left.+\left(\begin{array}{ccc}
\lambda_{m} & 0 & 0 \\
0 & 0 & 0 \\
0 & 0 & 0
\end{array}\right)\right]\left(\begin{array}{c}
\nu_{e} \\
\nu_{\mu} \\
\nu_{\tau}
\end{array}\right) .
\end{aligned}
$$

Here, $U$ is the Pontecorvo-Maki-Nakagawa-Sakata matrix $[119,120], \delta m^{2} \equiv m_{2}^{2}-m_{1}^{2}$, positive by convention, is the solar mass-squared difference and $\Delta m^{2} \equiv$ $\left|m_{3}^{2}-\left(m_{1}^{2}+m_{2}^{2}\right) / 2\right|$ is the atmospheric mass-squared difference. The sign \pm on $\Delta m^{2}$ corresponds to the mass hierarchy, with $(+)$ for $\mathrm{NH}$ and $(-)$ for $\mathrm{IH}$. In our main analysis we use the best-fit values from $[89,90]$ for the oscillation parameters. The matter-induced potential 
$[121,122]$ is given by $\lambda_{m}=\sqrt{2} G_{F}\left(n_{e^{-}}-n_{e^{+}}\right)$, where $n_{e^{ \pm}}$is the $e^{ \pm}$density in the medium. For antineutrinos one replaces $\lambda_{m} \rightarrow-\lambda_{m}$. In the limit $\left|\lambda_{m}\right| \gg \Delta m^{2} / 2 E$ the electron and anti-electron neutrino states are propagation eigenstates. This limit holds very well in the emission region of CCSN neutrinos $(r \lesssim 100 \mathrm{~km})$, where $\lambda_{m} \gtrsim$ $10^{5} \mathrm{~km}^{-1}$ and $\Delta m^{2} / 2 E \lesssim 1 \mathrm{~km}^{-1}$.

In a region with smoothly varying matter density, and away from resonances of Eq. (C1), an adiabatic limit applies in which a neutrino prepared in a propagation eigenstate will remain in it. When the adiabatic limit does not apply, probability leaks into other states. Since $\Delta m^{2} \gg \delta m^{2}$, one can factor the analysis into two regimes, where in each regime there is a pair of states which may approach a resonance region and a third decoupled state which remains far from resonance. Defining

$$
\omega_{H}=\frac{\Delta m^{2}}{2 E}, \quad \omega_{L}=\frac{\delta m^{2}}{2 E},
$$

the region where $\omega_{H} \sim \lambda_{m}$ is known as the $H$ resonance, and the region where $\omega_{L} \sim \lambda_{m}$ the $L$ resonance. In an effective two-level system with mixing angle $\theta_{t}$ and mass difference $\Delta m_{t}^{2}$, the degree of adiabaticity can be analyzed using the adiabaticity parameter $\gamma$, defined by

$$
\gamma=\frac{\Delta m_{t}^{2} \sin ^{2} 2 \theta_{t}}{2 E \cos 2 \theta_{t}\left|\frac{1}{\lambda_{m}} \frac{d \lambda_{m}}{d r}\right|} .
$$

The crossing probability for a neutrino traversing the resonance region is given by

$$
P \approx \exp \left(-\frac{\pi}{2} \gamma F\right)
$$

where $F$ is an $\mathcal{O}(1)$ parameter that depends on the matter distribution [123].

Shock fronts present a singular density profile, requiring special treatment using an abrupt approximation. The crossing probability when passing through a shock front is given by [123]

$$
P=\sin ^{2}\left(\theta_{m}^{+}-\theta_{m}^{-}\right),
$$

where $\theta_{m}$ denotes the effective mixing angle in matter and the superscript $+(-)$ denotes the mixing angle after (before) crossing the shock. In a 2-flavor case, the effective mixing angle is given by

$$
\tan 2 \theta_{m}=\frac{\sin 2 \theta}{\cos 2 \theta-2 E \lambda_{m} / \Delta m^{2}} .
$$

In the general 3-flavor case, the amplitude for crossing between different propagation eigenstates is given by

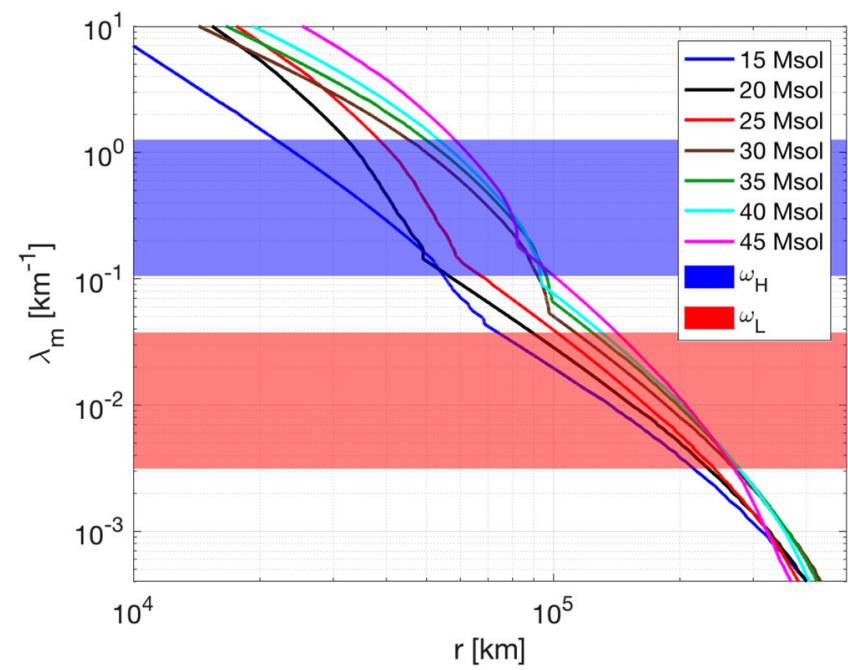

FIG. 17. Matter-induced potential $\lambda_{m}$ for stellar profiles calculated with MESA. Each curve corresponds to a progenitor, distinguished by its ZAMS mass. The shaded areas are $\omega_{H}$ and $\omega_{L}$, as defined in the text. For the width of the shaded area we set $E \in[5,60] \mathrm{MeV}$.

$$
\operatorname{Amp}\left(\nu_{i}^{m(-)} \rightarrow \nu_{j}^{m(+)}\right)=\left[U^{m(-) \dagger} U^{m(+)}\right]_{i j}
$$

such that the crossing probability is

$$
P\left(\nu_{i}^{m(-)} \rightarrow \nu_{j}^{m(+)}\right)=\left|\left[U^{m(-) \dagger} U^{m(+)}\right]_{i j}\right|^{2} .
$$

In practice we find $U^{m}(r)$ by diagonalizing the right-hand side of Eq. (C1).

\section{Resonance region analysis with MESA}

We now investigate the adiabaticity parameter $\gamma$ during the first few seconds of the CCSN. The first tool we employ is a set of nonrotating precollapse progenitor stellar profiles, calculated by Roni Waldman using the MESA stellar evolution code [124]. ${ }^{14}$ The zero-age main-sequence (ZAMS) stellar masses vary from 15 to $45 M_{\odot}$. The masses at the time of collapse vary nonmonotonically between $\approx 13$ to $24.4 M_{\odot}$ due to mass loss by winds. In Fig. 17 we plot the matter-induced potential $\lambda_{m}$ for the MESA profiles, and compare it with the vacuum inverse-oscillation wavelengths $\omega_{L}$ and $\omega_{H}$.

Figure 17 gives a stationary picture of the resonance regions before core-collapse. However, in the first few seconds of the collapse, this picture is sufficient for the analysis of flavor conversion in the CITE scenario. The reason for this is that for all of the profiles in Fig. 17, resonance regions $\left(\omega \sim \lambda_{m}\right)$ begin only at $r \gtrsim 10^{4} \mathrm{~km}$. When collapse starts, a shell located initially at radius $r_{0}$

\footnotetext{
${ }^{14}$ We thank Doron Kushnir for providing us with the MESA profiles.
} 
in the envelope of the star starts collapsing when the rarefaction wave reaches it, in a time [24]

$$
t_{\text {fall }}\left(r_{0}\right) \approx \frac{\pi r_{0}^{\frac{3}{2}}}{\sqrt{8 G M\left(r_{0}\right)}} .
$$

Using the MESA output and plugging for $r$ the first occurrence of the $(H)$ resonance, we find a minimal time for the $15 M_{\odot}$ progenitor of $t_{\text {fall }} \approx 6 \mathrm{sec}$. Thus, Fig. 17 shows the correct structure of the resonance regions during the first few seconds after core-collapse.

Evaluating Eq. (C3) for the MESA profiles, we find $\gamma>100$ in both of the $\omega_{L, H}$ resonance bands, except at composition jumps, which are visible in Fig. 17 mostly in the $\omega_{H}$ band. Treating these jumps in the abrupt approximation [as outlined in Eqs. (C5)-(C8)] may result in crossing probability $P_{\bar{H}} \sim \mathcal{O}(0.1)$ [therefore somewhat affecting the IH prediction for the IBD channel, cf. Eq. (10)]. Direct numerical integration of Eq. (C1) with the MESA profiles does not show any significant crossing probability. Composition jumps deserve a more thorough analysis, taking into account the detailed physical conditions in these regions. Such analysis, beyond the scope of the current work, is needed in order to verify that indeed composition jumps do not alter the adiabatic MikheyevSmirnov-Wolfenstein prediction. We conclude that matterinduced flavor conversion in the CITE scenario is most likely adiabatic, supporting our choice of $P_{L}=P_{H}=0$ in Sec. IV.

\section{PNS accretion shock}

We complement the stationary MESA analysis with a dynamical study of the PNS accretion shock during the first $1 \mathrm{sec}$ from core-collapse, using GR1D. In Fig. 18 we plot the matter-induced potential and the resonance bands for different post-bounce times in the core collapse simulation of a $15 M_{\odot}$ star. The PNS accretion shock is seen as a sharp density drop, receding inwards slightly from $r \gtrsim$ $100 \mathrm{~km}$ at $t_{\mathrm{PB}}=0.1 \mathrm{sec}$ to $r \sim 50 \mathrm{~km}$ at $t_{\mathrm{PB}}=0.68 \mathrm{sec}$. The shock occurs far from resonance, at $\lambda_{m} / \omega_{H} \gtrsim 10^{4}$. Equation (C5) then leads to negligible crossing probability, $P_{L}<P_{H}=\mathcal{O}\left(\omega_{H}^{2} / \lambda_{m}^{2}\right)$.

\section{Rotation-induced accretion shock}

References [24-26] found that successful CITE requires rotating stars. Rotation was needed to produce a

\footnotetext{
${ }^{15}$ This timing analysis can be refined by integrating the speed of sound $c_{s}$ from MESA simulations, $t_{\text {fall }}(r)=\int_{0}^{r} c_{s}^{-1}\left(r^{\prime}\right) d r^{\prime}$. This agrees with Eq. (C9) to $10 \%$ accuracy.

${ }^{16}$ Note that $t_{\text {fall }}$ is measured from the onset of core-collapse, when the rarefaction wave heads out towards the envelope, and not from core-bounce (when the $\bar{\nu}_{e}$ burst begins). Expressed as postbounce time, we should subtract the homologous corecollapse time so that $t_{\text {fall, } \mathrm{PB}} \approx t_{\text {fall }}-(0.3 \mathrm{sec})$. This small correction does not affect the results in this section.
}

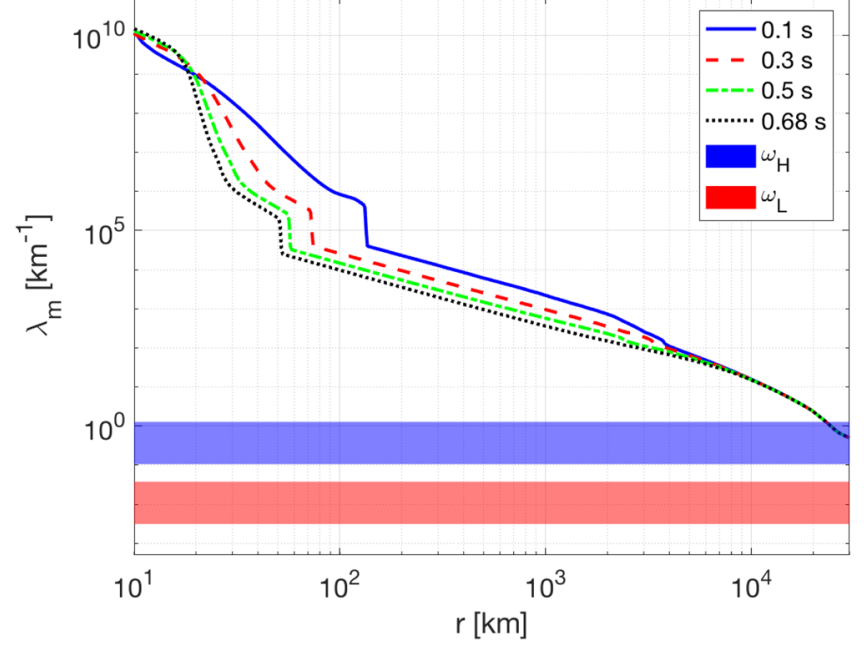

FIG. 18. Matter-induced potentials at different times based on the output of a single GR1D simulation of a $15 M_{\odot}$ progenitor with SLY4 nuclear equation of state. Listed times are postbounce. The shaded areas are $\omega_{H}$ and $\omega_{L}$, as defined in the text. We use $E \in[5,60] \mathrm{MeV}$. The steplike feature at $r \sim 100 \mathrm{~km}$ is the stalled bounce-shock. Since it is high above the resonance bands, any crossing probability is extremely negligible.

rotation-induced accretion shock (RIAS), which propagates outward through the infalling material and ignites the thermonuclear detonation once it encounters a mixed O-He layer. The RIAS is formed during the collapse of the envelope, when a rotating mass shell hits a centrifugal barrier. At the base of the RIAS, an accretion disk forms where the matter density is compressed and heated. Accretion disk neutrino emission in this scenario was studied in Ref. [35] using 2D numerical simulations.

CITE appears not to be very sensitive to the precise time, $t_{\text {disk }}$, at which the RIAS is launched. Reference [25] found strong explosions with $t_{\text {disk }}>10 \mathrm{sec}$, but in [35] CITE was demonstrated to operate also with $t_{\text {disk }} \sim 5 \mathrm{sec}$. We do not know if, e.g., $t_{\text {disk }} \sim 1 \mathrm{sec}$ would be consistent with a successful SN in CITE.

If $t_{\text {disk }}$ is larger than a few seconds, then the continued accretion onto the PNS leads to $\mathrm{BH}$ formation on a time $t_{\mathrm{BH}}<t_{\text {disk }}$. BH formation abruptly halts (within a dynamical time $\sim \mathrm{ms}$ ) the neutrino emission at $t_{\mathrm{BH}}$. The neutrino burst can be rekindled later on, with reduced but potentially observable luminosity, at $t=t_{\text {disk }}$. Analyzing the neutrino burst of SN1987A, Ref. [35] showed that an abrupt break in the neutrino light curve at $t_{\mathrm{BH}} \approx 2.7 \mathrm{sec}$, followed by accretion disk luminosity starting at $t_{\text {disk }} \approx 5 \mathrm{sec}$, is consistent with SN1987A data.

However, as discussed above, low $t_{\text {disk }} \sim 1$ sec cannot be excluded on theoretical grounds at this point. In this case, disk formation may precede $\mathrm{BH}$ formation, $t_{\text {disk }}<t_{\mathrm{BH}}$. If that happens, then detectable neutrino emission from the disk may continue across $t>t_{\mathrm{BH}}$ without 
being completely cutoff by the $\mathrm{BH}$ formation, smoothing out the spectacular signal of $\mathrm{BH}$ formation promised for $t_{\text {disk }}>t_{\mathrm{BH}}$. We now estimate the impact of disk formation for neutrino flavor conversion, relevant for $t_{\mathrm{disk}}<t_{\mathrm{BH}}$. Again, the important question to address is the level of adiabaticity for neutrino propagation.

Consider a rotating star, with the rotation on the $z=0$ plane parametrized by a function $f(r)$, specifying the ratio between the centrifugal force and the gravitational force. We assume $f \ll 1$. Once collapse begins, a shell starting at radius $r_{0}$ begins to fall at time $t_{\text {fall }}$ given by Eq. (C9). If no shock meets it, the shell hits a centrifugal barrier when it attains a radius $r_{\text {disk }}=(f / 2) r_{0}$. To lowest order in $f$, this occurs at a time

$$
t_{\text {disk }} \approx 2 t_{\text {fall }},
$$

independent of $f$. Just upstream of $r_{\text {disk }}$ the matter density is compressed by the factor

$$
\frac{\rho\left(r_{\text {disk }}\right)}{\rho_{0}\left(r_{0}\right)} \approx \frac{8 \sqrt{2}}{\pi \gamma_{\rho}\left(r_{0}\right)} f^{-\frac{3}{2}},
$$

where $\rho_{0}(r)$ is the precollapse density at $r_{0}$ and $\gamma_{\rho}=3-\frac{d \log M}{d \log r}$. [For $\rho_{0}(r) \propto r^{-\Gamma}$, for example, we have $\gamma_{\rho}=\Gamma$.] For reasonable values of $f$ between 0.01 to 0.1 and $\gamma_{\rho}$ between 1 to 3, Eq. (C11) gives a compression factor $\rho\left(r_{\text {disk }}\right) / \rho_{0}\left(r_{0}\right)$ ranging between 40 to 3000 .

Referring back to Fig. 17 and the discussion around Eq. (C9), and noting that the precollapse matter potential $\lambda_{m}$ in Fig. 17, when evaluated in the disk region at $t_{\text {disk }}$ during the collapse, is enhanced by the compression factor Eq. (C11), we conclude that if an accretion disk forms at $t_{\text {disk }}<5 \mathrm{sec}$, then it is located in a deep adiabatic region for neutrino propagation, $\lambda_{m} \gg \omega_{H}, \omega_{L}$.

\section{APPENDIX D: SOME COMMENTS ABOUT THE EFFECT OF ROTATION}

The GR1D simulations in this paper were made with nonrotating progenitor stars. Since CITE requires some rotation, it is natural to ask if this could affect the neutrino burst. Rotation in precollapse massive stars is not well understood [125-127], so we cannot give a definitive answer. In addition, as noted in Sec. C 3, a systematic study to assess the range of rotation profiles compatible with CITE is still lacking. However, as we now show, precollapse core rotation is likely low enough as to not significantly affect the results in the first few seconds postbounce.

To demonstrate this point, we consider a precollapse rotation profile similar to that used in the CITE simulation of Ref. [35]. This profile had $f=0.02$ at $r>1.2 \times 10^{4} \mathrm{~km}$

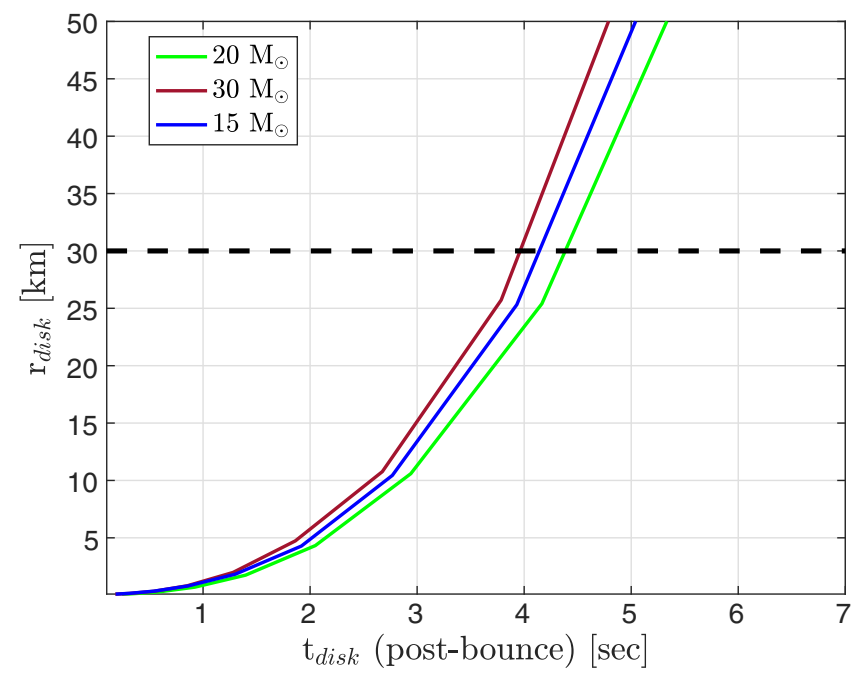

FIG. 19. The Keplerian disk formation radius as function of time postbounce. Note that the disk does not form in practice, because the free-fall is inhibited by the PNS accretion shock at $r_{\text {shock }} \gtrsim 50 \mathrm{~km}$. The dashed line marks the approximate location of the $\bar{\nu}_{e}$ neutrinosphere.

(in the ballpark of previous and subsequent analyses [43-45,125,128], and of the larger suit of CITE simulations studied in [25]). We extend the rotation inward ${ }^{17}$ imposing that the core at $r<1.2 \times 10^{4} \mathrm{~km}$ follows rigid body rotation, $f(r) \propto r^{3} / M(r)$. Using the calculations in Sec. C 3 [see Eq. (C10) and discussion around it], we apply the rotation profile $f(r)$ to the $(15,20,30) M_{\odot}$ progenitor stars used in the GR1D simulations in the body of this paper and calculate, for each progenitor, the location $r_{\text {disk }}$ of Keplerian centrifugal barrier formation for each precollapse mass element, as function of the postbounce time $t_{\text {disk }}$ at which that barrier would be attained.

The result of this exercise is shown in Fig. 19. To highlight the neutrino emission region, the dashed horizontal line marks the approximate location of the $\bar{\nu}_{e}$ neutrinosphere. Optically-thin accretion-dominated $\nu_{e}, \bar{\nu}_{e}$ emission extends between $r \sim 30-50 \mathrm{~km}$ (see Sec. II). Note that at early times $\left(t_{\text {disk }} \lesssim 5 \mathrm{sec}\right)$, the Keplerian disk does not form in practice: instead, the free-fall is inhibited by the PNS accretion shock at $r_{\text {shock }} \gtrsim 50 \mathrm{~km}$. Thus, what Fig. 19 demonstrates is that during the first few seconds postbounce centrifugal forces are unimportant for the dynamics of the shocked region above the PNS, where the neutrino emission is generated. For example, at $t_{\mathrm{PB}}<3 \mathrm{sec}\left(t_{\mathrm{PB}}<2 \mathrm{sec}\right)$, the centrifugal force acting on the matter crossing through the PNS accretion shock is less than $\sim 20 \%$ $(\sim 10 \%)$ of the gravitational force. During the first second postbounce the effect is negligibly small.

\footnotetext{
${ }^{17}$ For simplicity, Ref. [35] took $f=0$ below $1.2 \times 10^{4} \mathrm{~km}$.
} 
At late times-beyond what was directly analyzed in this work-once the accretion disk does form, as long as the disk is not optically thick to neutrinos the luminosity has been shown to be dominated by $\nu_{e, \bar{e}}$ from pair conversion and $e^{ \pm}$annihilation (see, e.g., [43-45]). We did not simulate this phase of the neutrino burst in the current work (although, see [35]), however, based on the arguments above we conclude that the CITE scenario predicts $\nu_{e, \bar{e}}$ dominance at the source throughout the burst.
[1] H. A. Bethe and J. R. Wilson, Astrophys. J. 295, 14 (1985).

[2] K. Kotake, K. Sato, and K. Takahashi, Rep. Prog. Phys. 69, 971 (2006).

[3] A. Burrows, Rev. Mod. Phys. 85, 245 (2013).

[4] H. T. Janka, T. Melson, and A. Summa, Annu. Rev. Nucl. Part. Sci. 66, 341 (2016).

[5] E. O'Connor et al., J. Phys. G 45, 104001 (2018).

[6] K. Nakamura, T. Takiwaki, T. Kuroda, and K. Kotake, Publ. Astron. Soc. Jpn. 67, 107 (2015).

[7] S. Seadrow, A. Burrows, D. Vartanyan, D. Radice, and M. A. Skinner, arXiv:1804.00689.

[8] I. Tamborra, G. Raffelt, F. Hanke, H.-T. Janka, and B. Mueller, Phys. Rev. D 90, 045032 (2014).

[9] D. Vartanyan, A. Burrows, D. Radice, A. Skinner, and J. Dolence, Mon. Not. R. Astron. Soc. 482, 351 (2019).

[10] S. M. Couch, E. Chatzopoulos, W. D. Arnett, and F. X. Timmes, Astrophys. J. 808, L21 (2015).

[11] B. Müller, M. Viallet, A. Heger, and H.-T. Janka, Astrophys. J. 833, 124 (2016).

[12] D. Vartanyan, A. Burrows, D. Radice, M. A. Skinner, and J. Dolence, Mon. Not. R. Astron. Soc. 477, 3091 (2018).

[13] H.-T. Janka, Annu. Rev. Nucl. Part. Sci. 62, 407 (2012).

[14] J. M. LeBlanc and J. R. Wilson, Astrophys. J. 161, 541 (1970).

[15] N. V. Ardelyan, G. S. Bisnovatyi-Kogan, and Y. P. Popov, Sov. Astron. 23, 705 (1979).

[16] N. V. Ardeljan, G. S. Bisnovatyi-Kogan, and S. G. Moiseenko, Mon. Not. R. Astron. Soc. 359, 333 (2005).

[17] A. Burrows, L. Dessart, E. Livne, C. D. Ott, and J. Murphy, Astrophys. J. 664, 416 (2007).

[18] T. Takiwaki and K. Kotake, Astrophys. J. 743, 30 (2011).

[19] A. Burrows, E. Livne, L. Dessart, C. Ott, and J. Murphy, Astrophys. J. 640, 878 (2006).

[20] A. Gilkis, N. Soker, and O. Papish, Astrophys. J. 826, 178 (2016).

[21] M. E. Burbidge, G. R. Burbidge, W. A. Fowler, and F. Hoyle, Rev. Mod. Phys. 29, 547 (1957).

[22] F. Hoyle and W. A. Fowler, Astrophys. J. 132, 565 (1960); 134, 1028(E) (1961).

[23] W. A. Fowler and F. Hoyle, Astrophys. J. Suppl. Ser. 9, 201 (1964).

[24] D. Kushnir and B. Katz, Astrophys. J. 811, 97 (2015).

[25] D. Kushnir, arXiv:1502.03111.

[26] D. Kushnir, arXiv:1506.02655.

[27] R. A. Gofman, A. Gilkis, and N. Soker, Mon. Not. R. Astron. Soc. 478, 703 (2018).

[28] K. Hirata et al. (Kamiokande-II Collaboration), Phys. Rev. Lett. 58, 1490 (1987).
[29] R. M. Bionta et al., Phys. Rev. Lett. 58, 1494 (1987).

[30] D. N. Spergel, T. Piran, A. Loeb, J. Goodman, and J. N. Bahcall, Science 237, 1471 (1987).

[31] J. M. Lattimer and A. Yahil, Astrophys. J. 340, 426 (1989).

[32] T. J. Loredo and D. Q. Lamb, Phys. Rev. D 65, 063002 (2002).

[33] G. Pagliaroli, F. Vissani, M. L. Costantini, and A. Ianni, Astropart. Phys. 31, 163 (2009).

[34] F. Vissani, J. Phys. G 42, 013001 (2015).

[35] K. Blum and D. Kushnir, Astrophys. J. 828, 31 (2016).

[36] S. M. Adams, C. S. Kochanek, J. F. Beacom, M. R. Vagins, and K. Z. Stanek, Astrophys. J. 778, 164 (2013).

[37] A. Mirizzi, I. Tamborra, H.-T. Janka, N. Saviano, K. Scholberg, R. Bollig, L. Hudepohl, and S. Chakraborty, Riv. Nuovo Cimento 39, 1 (2016).

[38] S. Horiuchi and J. P. Kneller, arXiv:1709.01515.

[39] E. O'Connor and C. D. Ott, Astrophys. J. 730, 70 (2011).

[40] S. E. Woosley, A. Heger, and T. A. Weaver, Rev. Mod. Phys. 74, 1015 (2002).

[41] T. Sukhbold and S. Woosley, Astrophys. J. 783, 10 (2014).

[42] K. J. Borkowski and S. P. Reynolds, Astrophys. J. 846, 13 (2017).

[43] R. Popham, S. E. Woosley, and C. Fryer, Astrophys. J. 518, 356 (1999).

[44] A. MacFadyen and S. E. Woosley, Astrophys. J. 524, 262 (1999).

[45] T. Liu, B. Zhang, Y. Li, R.-Y. Ma, and L. Xue, Phys. Rev. D 93, 123004 (2016).

[46] F. An et al. (JUNO Collaboration), J. Phys. G 43, 030401 (2016).

[47] M. Wurm et al., Acta Phys. Pol. B 41, 1749 (2010).

[48] S. Chakraborty, T. Fischer, A. Mirizzi, N. Saviano, and R. Tomas, Phys. Rev. Lett. 107, 151101 (2011).

[49] S. Sarikas, G. G. Raffelt, L. Hudepohl, and H.-T. Janka, Phys. Rev. Lett. 108, 061101 (2012).

[50] S. Chakraborty, A. Mirizzi, N. Saviano, and D.d. S. Seixas, Phys. Rev. D 89, 093001 (2014).

[51] G. Pagliaroli, C. Lujan-Peschard, M. Mitra, and F. Vissani, Phys. Rev. Lett. 111, 022001 (2013).

[52] J. F. Beacom, W. M. Farr, and P. Vogel, Phys. Rev. D 66, 033001 (2002).

[53] B. Dasgupta and J. F. Beacom, Phys. Rev. D 83, 113006 (2011).

[54] R. Laha and J. F. Beacom, Phys. Rev. D 89, 063007 (2014).

[55] R. Laha, J. F. Beacom, and S. K. Agarwalla, arXiv:1412 .8425 .

[56] A. Nikrant, R. Laha, and S. Horiuchi, arXiv:1711.00008. 
[57] H. Minakata, H. Nunokawa, R. Tomas, and J. W. F. Valle, Phys. Lett. B 542, 239 (2002).

[58] V. Barger, D. Marfatia, and B. P. Wood, Phys. Lett. B 547, 37 (2002).

[59] H. Minakata, H. Nunokawa, R. Tomas, and J. W. F. Valle, J. Cosmol. Astropart. Phys. 12 (2008) 006.

[60] A. Gallo Rosso, F. Vissani, and M. C. Volpe, J. Cosmol. Astropart. Phys. 11 (2017) 036.

[61] A. Gallo Rosso, F. Vissani, and M. C. Volpe, J. Cosmol. Astropart. Phys. 04 (2018) 040.

[62] J.-S. Lu, Y.-F. Li, and S. Zhou, Phys. Rev. D 94, 023006 (2016).

[63] S. Horiuchi, K. Nakamura, T. Takiwaki, and K. Kotake, J. Phys. G 44, 114001 (2017).

[64] Y. Fukuda et al. (Super-Kamiokande Collaboration), Nucl. Instrum. Methods Phys. Res., Sect. A 501, 418 (2003).

[65] K. Abe et al. (Hyper-Kamiokande Collaboration), arXiv: 1805.04163.

[66] R. Acciarri et al. (DUNE Collaboration), arXiv:1601 .02984 .

[67] A. Burrows, D. Vartanyan, J. C. Dolence, M. A. Skinner, and D. Radice, Space Sci. Rev. 214, 33 (2018).

[68] D. Radice, A. Burrows, D. Vartanyan, M. A. Skinner, and J. C. Dolence, Astrophys. J. 850, 43 (2017).

[69] E. O'Connor and C. D. Ott, Classical Quantum Gravity 27, 114103 (2010).

[70] E. O’Connor, Astrophys. J. Suppl. Ser. 219, 24 (2015).

[71] E. Chabanat, P. Bonche, P. Haensel, J. Meyer, and R. Schaeffer, Nucl. Phys. A635, 231 (1998); A643, 441 (1998).

[72] A. S. Schneider, L. F. Roberts, and C. D. Ott, Phys. Rev. C 96, 065802 (2017).

[73] J. M. Lattimer and F. D. Swesty, Nucl. Phys. A535, 331 (1991).

[74] B. K. Agrawal, S. Shlomo, and V. K. Au, Phys. Rev. C 72, 014310 (2005).

[75] A. Perego, M. Hempel, C. Frhlich, K. Ebinger, M. Eichler, J. Casanova, M. Liebendoerfer, and F. K. Thielemann, Astrophys. J. 806, 275 (2015).

[76] A. Nikrant, R. Laha, and S. Horiuchi, Phys. Rev. D 97, 023019 (2018).

[77] J.B. Birks, The Theory and Practice of Scintillation Counting (Mcmillan, 1964), http://www.slac.stanford .edu/spires/find/books/www?cl=QCD928:B52.

[78] B. von Krosigk, L. Neumann, R. Nolte, S. Rottger, and K. Zuber, Eur. Phys. J. C 73, 2390 (2013).

[79] L. A. Ahrens et al., Phys. Rev. D 35, 785 (1987).

[80] C. A. Aidala, S. D. Bass, D. Hasch, and G. K. Mallot, Rev. Mod. Phys. 85, 655 (2013).

[81] R. S. Sufian, K.-F. Liu, and D. G. Richards, arXiv:1809 .03509 .

[82] K. Nakazato, T. Suzuki, and M. Sakuda, Prog. Theor. Exp. Phys. 2018, 123E02 (2018).

[83] L. Wolfenstein, Phys. Rev. D 17, 2369 (1978).

[84] S. P. Mikheyev and A. Yu. Smirnov, Sov. J. Nucl. Phys. 42, 913 (1985).

[85] A. S. Dighe and A. Yu. Smirnov, Phys. Rev. D 62, 033007 (2000).

[86] S. Horiuchi and J. P. Kneller, J. Phys. G 45, 043002 (2018).
[87] S. Chakraborty, A. Mirizzi, and G. Sigl, Phys. Rev. D 87, 017302 (2013).

[88] S. Seadrow, A. Burrows, D. Vartanyan, D. Radice, and M. A. Skinner, Mon. Not. R. Astron. Soc. 480, 4710 (2018).

[89] I. Esteban, M. C. Gonzalez-Garcia, M. Maltoni, I. Martinez-Soler, and T. Schwetz, J. High Energy Phys. 01 (2017) 087.

[90] Nufit 3.2 (2018), www.nu-fit.org.

[91] H. Duan, G. M. Fuller, and Y.-Z. Qian, Annu. Rev. Nucl. Part. Sci. 60, 569 (2010).

[92] H. Duan, G. M. Fuller, J. Carlson, and Y.-Z. Qian, Phys. Rev. D 74, 105014 (2006).

[93] G. L. Fogli, E. Lisi, A. Marrone, and A. Mirizzi, J. Cosmol. Astropart. Phys. 12 (2007) 010.

[94] G. G. Raffelt and A. Yu. Smirnov, Phys. Rev. D 76, 081301 (2007); 77, 029903 (2008).

[95] G. L. Fogli, E. Lisi, A. Marrone, A. Mirizzi, and I. Tamborra, Phys. Rev. D 78, 097301 (2008).

[96] B. Dasgupta, A. Dighe, G. G. Raffelt, and A. Yu. Smirnov, Phys. Rev. Lett. 103, 051105 (2009).

[97] G. Fogli, E. Lisi, A. Marrone, and I. Tamborra, J. Cosmol. Astropart. Phys. 04 (2009) 030.

[98] B. Dasgupta, A. Mirizzi, I. Tamborra, and R. Tomas, Phys. Rev. D 81, 093008 (2010).

[99] A. Esteban-Pretel, A. Mirizzi, S. Pastor, R. Tomas, G. G. Raffelt, P. D. Serpico, and G. Sigl, Phys. Rev. D 78, 085012 (2008).

[100] S. Sarikas, G. G. Raffelt, L. Hudepohl, and H.-T. Janka, Phys. Rev. Lett. 108, 061101 (2012).

[101] N. Saviano, S. Chakraborty, T. Fischer, and A. Mirizzi, Phys. Rev. D 85, 113002 (2012).

[102] J. F. Cherry, J. Carlson, A. Friedland, G. M. Fuller, and A. Vlasenko, Phys. Rev. Lett. 108, 261104 (2012).

[103] S. Sarikas, I. Tamborra, G. Raffelt, L. Hudepohl, and H.-T. Janka, Phys. Rev. D 85, 113007 (2012).

[104] S. Sarikas, D. d. S. Seixas, and G. Raffelt, Phys. Rev. D 86, 125020 (2012).

[105] G. Raffelt and D.d. S. Seixas, Phys. Rev. D 88, 045031 (2013).

[106] R. S. Hansen and S. Hannestad, Phys. Rev. D 90, 025009 (2014).

[107] A. Mirizzi, Phys. Rev. D 88, 073004 (2013).

[108] S. Chakraborty and A. Mirizzi, Phys. Rev. D 90, 033004 (2014).

[109] G. Mangano, A. Mirizzi, and N. Saviano, Phys. Rev. D 89, 073017 (2014).

[110] C. Lunardini and A. Yu. Smirnov, Nucl. Phys. B616, 307 (2001).

[111] J. F. Beacom, Annu. Rev. Nucl. Part. Sci. 60, 439 (2010).

[112] R. B. Patterson, Annu. Rev. Nucl. Part. Sci. 65, 177 (2015).

[113] A. Ianni, G. Pagliaroli, A. Strumia, F. R. Torres, F. L. Villante, and F. Vissani, Phys. Rev. D 80, 043007 (2009).

[114] S. M. Bilenky and J. Hosek, Phys. Rep. 90, 73 (1982).

[115] M. Tanabashi et al. (Particle Data Group). Phys. Rev. D 98, 030001 (2018).

[116] A. Strumia and F. Vissani, Phys. Lett. B 564, 42 (2003).

[117] W. J. Marciano and Z. Parsa, J. Phys. G 29, 2629 (2003). 
[118] M. C. Gonzalez-Garcia and Y. Nir, Rev. Mod. Phys. 75, 345 (2003).

[119] Z. Maki, M. Nakagawa, and S. Sakata, Prog. Theor. Phys. 28, 870 (1962).

[120] M. Kobayashi and T. Maskawa, Prog. Theor. Phys. 49, 652 (1973).

[121] L. Wolfenstein, Phys. Rev. D 17, 2369 (1978).

[122] S. P. Mikheev and A. Yu. Smirnov, Nuovo Cimento C9, 17 (1986).

[123] T.-K. Kuo and J. T. Pantaleone, Rev. Mod. Phys. 61, 937 (1989).
[124] B. Paxton, L. Bildsten, A. Dotter, F. Herwig, P. Lesaffre, and F. Timmes, Astrophys. J. Suppl. Ser. 192, 3 (2011).

[125] A. Heger, N. Langer, and S. E. Woosley, Astrophys. J. 528, 368 (2000).

[126] A. Maeder and G. Meynet, Annu. Rev. Astron. Astrophys. 38, 143 (2000).

[127] G. Meynet, S. Ekstrom, A. Maeder, P. Eggenberger, H. Saio, V. Chomienne, and L. Haemmerle, Lect. Notes Phys. 865, 3 (2013).

[128] A. Heger, S. E. Woosley, N. Langer, and H. C. Spruit, IAU Symp. 215, 591 (2004). 